\title{
The $\alpha 1 \mathrm{~K} 276 \mathrm{E}$ Startle Disease Mutation Reveals Multiple Intermediate States in the Gating of Glycine Receptors
}

\author{
Remigijus Lape, ${ }^{1 \star}$ Andrew J. R. Plested, ${ }^{2,3 \star}$ Mirko Moroni, ${ }^{1}$ David Colquhoun, ${ }^{1}$ and Lucia G. Sivilotti ${ }^{1}$ \\ ${ }^{1}$ Department of Neuroscience, Physiology and Pharmacology, University College London, London WC1E 6BT, United Kingdom, ${ }^{2}$ Leibniz-Institut für \\ Molekulare Pharmakologie, Berlin 13125, Germany, and ${ }^{3}$ Cluster of Excellence NeuroCure, Charité-Universitätsmedizin Berlin, Berlin 10117, Germany
}

Loss-of-function mutations in human glycine receptors cause hyperekplexia, a rare inherited disease associated with an exaggerated startle response. We have studied a human disease mutation in the M2-M3 loop of the glycine receptor $\alpha 1$ subunit (K276E) using direct fitting of mechanisms to single-channel recordings with the program HJCFIT. Whole-cell recordings from HEK293 cells showed the mutation reduced the receptor glycine sensitivity. In single-channel recordings, rat homomeric $\alpha 1$ K276E receptors were barely active, even at $200 \mathrm{~mm}$ glycine. Coexpression of the $\beta$ subunit partially rescued channel function. Heteromeric mutant channels opened in brief bursts at $300 \mu \mathrm{m}$ glycine (a concentration that is near-maximal for wild type) and reached a maximum one-channel open probability of about $45 \%$ at $100 \mathrm{~mm}$ glycine (compared to $96 \%$ for wild type). Distributions of apparent open times contained more than one component in high glycine and, therefore, could not be described by mechanisms with only one fully liganded open state. Fits to the data were much better with mechanisms in which opening can also occur from more than one fully liganded intermediate (e.g., "primed" models). Brief pulses of glycine $(\sim 3 \mathrm{~ms}, 30 \mathrm{~mm})$ applied to mutant channels in outside-out patches activated currents with a slower rise time (1.5 ms) than those of wild-type channels $(0.2 \mathrm{~ms})$ and a much faster decay. These features were predicted reasonably well by the mechanisms obtained from fitting single-channel data. Our results show that, by slowing and impairing channel gating, the K276E mutation facilitates the detection of closed reaction intermediates in the activation pathway of glycine channels.

\section{Introduction}

Glycine receptors mediate IPSCs essential for motor control in the brainstem and spinal cord. Like other Cys-loop channels (Thompson et al., 2010), these receptors are formed by five subunits arranged in a pseudosymmetric ring around the central pore. Adult synaptic channels are mostly $\alpha 1 \beta$ heteromers, but $\alpha$ homomers are also functional. Heteromers have lower conductance, are less sensitive to picrotoxin block, and are clustered at synapses by gephyrin (Pribilla et al., 1992; Bormann et al., 1993; Meyer et al., 1995). The $\beta$ subunit is also taken (from shifts in glycine concentration response relations) to control glycine potency in heteromeric channels (Grudzinska et al., 2005).

In each subunit, the extracellular glycine-binding domains form a juxtamembrane interface with the membrane helices and the conserved M2-M3 loop, through which conformational changes induced by agonist binding propagate to the channel gate. In glycine receptors, mutations in the M2-M3 loop can strongly inhibit channel activation, and thus cause hyperekplexia

\footnotetext{
Received Aug. 24, 2011; revised Nov. 14, 2011; accepted Nov. 16, 2011.

Author contributions: R.L., A.J.R.P., and L.G.S. designed research; R.L., A.J.R.P., and M.M. performed research; R.L., A.J.R.P., and D.C. contributed unpublished reagents/analytic tools; R.L., A.J.R.P., M.M., D.C., and L.G.S. analyzed data; R.L., A.J.R.P., D.C., and L.G.S. wrote the paper.

This work was funded in part by the Medical Research Council (Programme Grant G0400869 to L.G.S.) and by the Deutsche Forschungsgemeinschaft (Exc 257 NeuroCure to A.J.R.P.). We thank Paul Groot-Kormelink for making the $\alpha 1$ K276E mutant.

${ }^{*}$ R.L. and A.J.R.P. contributed equally to this work.

Correspondence should be addressed to Lucia G. Sivilotti at the above address. E-mail: I.sivilotti@ucl.ac.uk.

M. Moroni's present address: Max Delbrück Center for Molecular Medicine, Berlin 13125, Germany.

DOI:10.1523/JNEUROSCI.4346-11.2012

Copyright $\odot 2012$ the authors $\quad 0270-6474 / 12 / 321336-17 \$ 15.00 / 0$
}

(Langosch et al., 1994; Chung et al., 2010). Our early work (Lewis et al., 1998) showed that one such hyperekplexia mutation in position $24^{\prime}, \alpha 1 \mathrm{~K} 276 \mathrm{E}$ (Elmslie et al., 1996), acts by impairing gating.

Over the past 10 years, evidence has emerged that the gating reaction in neurotransmitter receptors is not a simple isomerization from resting to open. Instead, receptors are likely to traverse a series of intermediate shut states before they open. These states can account for the efficacy of agonists in Cys-loop receptors, as well as the strong apparent cooperativity of wild-type heteromeric glycine channels (Burzomato et al., 2004; Lape et al., 2008; Sivilotti, 2010).

Unfortunately, in wild-type Cys-loop receptors, intermediates are so short-lived that most evidence of their existence is indirect. For example, mutations that cause strong shifts in the apparent efficacy of choline at muscle nicotinic receptors generally obey a linear free-energy relation (Grosman et al., 2000). This analysis suggests different parts of the receptor move at different times in a sequence of movements from early to late in the gating reaction (Chakrapani et al., 2004). The existence of several such gating steps has also been inferred from the complexity of dwelltime distributions observed for spontaneously opening gain-offunction mutants (first reported by Grosman, 2003) and for muscle nicotinic receptors whose moving parts have been trapped by introduced disulfide bonds (Mukhtasimova et al., 2009). Another approach, which we continue with this manuscript, is to attempt to detect intermediate shut states directly by including them explicitly in reaction mechanisms (Burzomato et al., 2004; Plested et al., 2007; Lape et al., 2008). Given single- 
channel recordings containing sufficient information, global maximum likelihood fitting allows us to examine the features needed by a postulated activation mechanism to describe complex features of equilibrium and nonequilibrium data (Colquhoun et al., 2003). Here, we exploit this method to characterize the gating impairment caused by the $\alpha 1 \mathrm{~K} 276 \mathrm{E}$ mutation and show that multiple intermediate shut states precede the opening of glycine channels.

\section{Materials and Methods}

Cell culture and molecular biology. Cell culture was performed as described previously (Plested et al., 2007). The K276E mutation was introduced in the rat $\alpha 1$ subunit (GenBank accession number AJ310834) with the QuikChange kit (Stratagene) and verified by full-length sequencing. HEK 293 cells were transfected by $\mathrm{Ca}^{2+}$ phosphate-DNA coprecipitation (Groot-Kormelink et al., 2002) using $3 \mu \mathrm{g}$ of plasmid DNA for each $35 \mathrm{~mm}$ dish, including $0.3 \mu \mathrm{g}$ of DNA coding for green fluorescent protein as a marker (EGFP-cl, Clontech). To express heteromeric channels, we included plasmid DNA coding for the $\beta$ subunit (GenBank accession number AJ310839) of the rat glycine receptor in a 20- to 40 -fold excess, to reduce homomer formation (Burzomato et al., 2003). At long intervals following transfection $(>48 \mathrm{~h})$, the single-channel properties of mutant glycine receptors can become heterogeneous (Plested et al., 2007). For this reason, recordings were made, at most, $36 \mathrm{~h}$ following transfection, even though this increased the proportion of patches that showed no activity. Glycine receptor activity appeared homogeneous, to the extent that we can detect, in these circumstances. All recordings were at room temperature $\left(21^{\circ} \mathrm{C}\right)$.

Whole-cell recording. Currents elicited by local U-tube application of $0.3-200 \mathrm{~mm}$ glycine were recorded at a holding potential of $-60 \mathrm{mV}$. Access resistance was never $>10 \mathrm{M} \Omega$ and was compensated by at least $80 \%$. The bath solution (for all recording configurations) was composed as follows (in mM): $\mathrm{NaCl}, 102.7 ; \mathrm{MgCl}_{2}, 1.2 ; \mathrm{CaCl}_{2}, 2 ; \mathrm{KCl}, 4.7$; glucose, 14; Na gluconate, 20 ; sucrose, 15 ; tetraethylammonium chloride (TEA-Cl), 20; and HEPES, 10, pH adjusted to 7.4 with $\mathrm{NaOH}$. The pipette solution contained the following (in $\mathrm{mm}$ ): $107.1 \mathrm{KCl}, 1 \mathrm{CaCl}_{2}, 1$ $\mathrm{MgCl}_{2}, 10$ HEPES, 11 EGTA, $2 \mathrm{MgATP}$, and 20 TEA-Cl. A full concentration-response curve was obtained in each cell. To control for the run-up of responses during the recording, peak currents from each cell were normalized to the current elicited by a standard concentration (typically $3 \mathrm{~mm}$ ) applied periodically during the experiment. The Hill equation was then fitted to each individual concentration-response curve, and values were normalized to the fitted maximum (program CVFIT; http://www.OneMol.org.uk). These renormalized responses were pooled and fitted with the Hill equation.

Single-channel recordings. For cell-attached recordings, the pipette solution was the appropriate mixture of the bath solution and a glycine stock [composition (in mM): glycine, $200 ; \mathrm{NaCl}, 36.7 ; \mathrm{MgCl}_{2}, 1.2 ; \mathrm{CaCl}_{2}$, 2; $\mathrm{KCl}, 4.7$; TEA-Cl, 20; and HEPES, 10; $\mathrm{pH}$ adjusted to 7.4 with $\mathrm{NaOH}$ ]. We found that the chloride reversal potential varied from cell to cell. This variance was the result of differences in internal chloride concentration, over which we have no control in the cell-attached configuration. Because of that, we hyperpolarized patches containing heteromeric receptors by applying a pipette voltage of $80-110 \mathrm{mV}$ to hold the single-channel current amplitude consistent in each patch at $\sim 3 \mathrm{pA}$ (Burzomato et al., 2004). This procedure controls for the minor effects of voltage on glycine receptor kinetics (Gill et al., 2006). Patch currents were prefiltered by the four-pole Bessel filter of the Axopatch 200B amplifier (MDS Analytical Technologies) at $10 \mathrm{kHz}$ and recorded to digital audio tape. For off-line analysis, single-channel data were filtered at $3-5 \mathrm{kHz}$ using an eight-pole Bessel filter (Frequency Devices) and digitized at $50 \mathrm{kHz}$ (CONSAM program, see http:// www.OneMol.org.uk). All chemicals were obtained from Sigma.

Analysis of single-channel records. Single-channel records were idealized by time course fitting (SCAN; Colquhoun and Sigworth, 1995) to obtain 7000-26,000 transitions per patch (mean 15,436). Low-noise recording techniques (Benndorf, 1995) enabled a signal-to-noise ratio as high as 20 ( $5 \mathrm{kHz}$ filter) for heteromeric receptors and thus the unambiguous detection of shut times as short as $30 \mu \mathrm{s}$ in patches recorded in high concentrations of glycine ( $\geq 3 \mathrm{~mm}$ ). However, at the lowest concentration used for recordings of heteromers $(300 \mu \mathrm{M})$, short shuttings were often interspersed with short openings. Filtering of adjacent brief openings and shuttings resulted in complex, ambiguous waveforms, and these events could not be reliably detected or measured. Isolated short openings from the baseline also became more frequent. The theoretical rate of false-event detection estimated from the data signal-to-noise ratio with Equation 17 (Colquhoun and Sigworth, 1995) was low, and we further reduced the potential for bias introduced by overfitting of groups of short events by imposing a conservative resolution of $40 \mu \mathrm{s}$ on sequences of heteromeric dwell times at all concentrations.

Suitability of patches for further analysis was checked first by examining the stability of amplitudes and of open and shut times (Colquhoun and Sigworth, 1995). As in previous studies (Beato et al., 2004; Burzomato et al., 2004), we observed only one conductance level in the amplitude histogram, which was well fitted with a single Gaussian function (Colquhoun and Sigworth, 1995). At this point, one patch was discarded because of inconsistencies in amplitudes, possibly arising from an interdigitated mixture of activations from both homomeric and heteromeric channels. Empirical fitting of idealized data (EKDIST program) was done to check the consistency of the distributions, to determine (from the shut time distributions) the critical times for dividing channel openings into groups, and to thus give us confidence that all openings in the group came from the same individual channel. This is the first step for maximum likelihood model fitting.

For recordings at the lowest concentration of glycine $(300 \mu \mathrm{M})$, activations of heteromeric receptors were clearly divisible into bursts by application of a critical shut time $\left(t_{\text {crit }}\right)$. It was not obvious, however, that the groups of openings were separated enough to ensure that each group consisted of an individual activation of the channel (see Results and Discussion). At glycine concentrations of $\geq 3 \mathrm{~mm}$, channel activations formed clear clusters separated by long quiescent periods (from $1 \mathrm{~s}$ to minutes). These clusters represent the continuous activity of a single channel [discussed in the article by Burzomato et al. (2004), on the basis of the study by Colquhoun and Hawkes (1990)] and were used to construct a single-channel open probability $\left(P_{\text {open }}\right)-$ concentration relation. The mean number of apparent open periods per cluster was 635, with the longest cluster consisting of 4938 apparent open periods. The probability of being open for each patch was estimated from the total open time within clusters divided by the total cluster duration (Burzomato et al., 2004; Plested et al., 2007). These values were averaged and fitted with the Hill equation (least-squares fit with weights from the SDs of the means at each concentration) using the program CVFIT. We used the Hill slope, $E C_{50}$ and maximum open probability $\left(P_{\max }\right)$ estimated from this analysis as one of the types of observables, together with dwell times and openshut correlations, for assessing the predictions of the maximum likelihood fits. The correlation plots provide a synoptic view of the (negative) correlation between open times and adjacent shut times, and they test the extent to which the observed correlations are predicted by the fit. Briefly, the mean apparent open time for openings adjacent to shut times in a number of contiguous ranges is plotted against the mean of the shut times in each range. The open times adjacent to short shuttings are, on average, longer than those adjacent to long shuttings (e.g., Fig. 9 in Colquhoun et al., 1996). The predictions are calculated with exact allowance for missed brief events.

The actual mechanisms that were fitted are not described by the Hill equation, and consequently their Hill slopes were not constant. For the purpose of comparing the open probability data with the results of the mechanism fits, the predicted Hill slope at the $E C_{50}\left(n_{\mathrm{H} 50}\right)$ was determined numerically for the latter using the following equation:

$$
n_{\mathrm{H} 50}=\left.\frac{\mathrm{d} \ln \left(\frac{P_{\text {open }}}{P_{\max }-P_{\text {open }}}\right)}{\mathrm{d} \ln (G)}\right|_{G=E C_{50}}
$$

where $G$ is the glycine concentration.

Maximum likelihood fitting of putative mechanisms was performed as described previously (HJCFIT program) (Colquhoun et al., 2003). At 300 $\mu \mathrm{M}$ glycine, the lowest glycine concentration where channel activity was observed, groups were treated as "bursts" defined by a critical shut time of 0.6 
or $1.2 \mathrm{~ms}$ and initial Colquhoun-Hawkes-Srodzinski vectors were used (Colquhoun et al., 1996) to recover some of the information from the interburst shut times (Colquhoun et al., 2003). At higher concentrations (3-100 $\mathrm{mm})$, the groups were divided into clusters by a critical shut time of $30-80$ $\mathrm{ms}$. Twelve patches recorded at five different concentrations $(0.3,3,10,30$, and $100 \mathrm{~mm}$ glycine) were grouped into three independent sets for simultaneous fitting with HJCFIT. Each set contained four patches, one at each glycine concentration: $0.3,3$ or 10,30 , and $100 \mathrm{~mm}$.

Concentration jumps: recording, analysis, and simulations. Macroscopic glycine-activated currents were recorded from outside-out patches, held at $-100 \mathrm{mV}$, using thick-walled borosilicate pipettes (Harvard Apparatus) fire polished to a final resistance of 6-10 $\mathrm{M} \Omega$. To mimic the low intracellular $\mathrm{Cl}^{-}$present in HEK cells, and reproduce the conditions experienced by channels in cell-attached patches (Pitt et al., 2008), we used a pipette solution with $30 \mathrm{~mm}$ total chloride, containing the following (in mM): $6 \mathrm{KCl}, 101 \mathrm{~K}$ gluconate, $1 \mathrm{CaCl}_{2}, 1 \mathrm{MgCl}_{2}, 10 \mathrm{HEPES}, 11$ EGTA, 20 TEA-Cl, and $2 \mathrm{MgATP}$. The $\mathrm{pH}$ was adjusted to 7.2 with $\mathrm{KOH}$, and the osmolarity was corrected to $310 \mathrm{mOsm}$ with sucrose. The junction potential was $9.5 \mathrm{mV}$ at $20^{\circ} \mathrm{C}$ (Clampex 9.2).

All concentration jumps were performed using a piezo-stepper (Burleigh Instruments) with an application tool made from theta tube glass (Hilgenberg) with final tip diameter of $150 \mu \mathrm{m}$. Voltage commands for the stepper were square pulses (2-3.5 ms, applied at $5 \mathrm{~s}$ intervals), conditioned by lowpass eight-pole Bessel filtering ( $-3 \mathrm{~dB}$ frequency $5 \mathrm{kHz}$ ) to reduce oscillations. The exchange time was estimated by recording the open-tip response to the application of diluted extracellular solution $\left(70 \% \mathrm{H}_{2} \mathrm{O}\right)$ after rupture of the patch. We used for analysis only patches for which the $20-80 \%$ exchange time was faster than $200 \mu \mathrm{s}$, and for which the decline in peak amplitude did not exceed 5\% during the run. Traces shown are averages of at least 10 individual agonist-activated currents. The time course of deactivation was fitted by least squares to the digitized points with two exponential components. Currents were filtered at $5 \mathrm{kHz}$, digitized at $50 \mathrm{kHz}$ with Digidata 1322A, and saved directly on computer with Clampex software (all MDS Analytical Technologies).

Several technical factors suggest caution in the interpretation of macroscopic currents. First, it is possible that channels have different properties in outside-out versus cell-attached patches. Second, in three of eight patches from cells expressing heteromeric K276E receptors, we detected a fast component of sag ("desensitization"; when measured from fits to responses to $500 \mathrm{~ms}$ applications of glycine, the time constant was $3.8 \pm 0.3 \mathrm{~ms} ; 50 \pm 10 \%$ relative amplitude). In one patch, this sag was apparent even during a $2 \mathrm{~ms}$ pulse. This phenomenon was absent in other patches. In wild-type receptors, the amplitude of this fast sag is correlated to the magnitude of the current (Pitt et al., 2008), suggesting it is an artifact related either to channel density effects on desensitization (Legendre et al., 2002) or to changes in chloride gradient (Moroni et al., 2011).

Calculations using mechanisms that included a fast desensitized state confirmed that fast sag can truncate the slow rising phase of the jump responses appreciably (data not shown). We exclude desensitized gaps from analysis of our single-channel records. Clusters had durations of seconds, rather than the roughly $10 \mathrm{~ms}$ duration expected if the fast sag represents receptor desensitization. Thus, it is unlikely that this sag is relevant to the channel activations in cell-attached configuration that we fitted by maximum likelihood. We therefore excluded from analysis macroscopic patch recordings where fast sag was pronounced enough to distort the rising phase of the glycine-activated current. Undetected variations in the extent of fast sag may explain the wide range of rise times we measured for mutant receptors.

We simulated concentration jumps using the Realistic Concentration Jump script (DC_PyPs repository, http://code.google.com/p/dc-pyps/). Briefly, concentration profiles were simulated with rising and falling phases described by an error function (Sachs, 1999). We evaluated the occupancy of the states in the mechanism at $8 \mu$ s intervals using the following equation (Colquhoun and Hawkes, 1977): $P(t+\delta t)=P(t) \cdot \exp \left[Q\left(c_{t}\right) \cdot \delta t\right]$, where $P(t)$ is the occupancy of the model at time $t, c_{t}$ is the concentration at time $t$, and $Q(c)$ is the $Q$ matrix at the given concentration of glycine, $c$.

We calculated responses to $0.5-12 \mathrm{~ms}$ glycine pulse profiles (with symmetrical $10-90 \%$ rise and fall times, varying from $0 \mu \mathrm{s}$ - a square pulse- to $750 \mu \mathrm{s}$ ) for $Q$-matrices obtained from fits to the three sets of single-channel data. Surprisingly, for the mechanisms tested, this range of pulse rise times did not greatly affect the shape of the simulated responses, except that the fastest component of the deactivation decay tended to be slower when glycine was removed more slowly. We therefore report here the values that correspond most closely to the likely solution exchange in patch recordings $(500 \mu \mathrm{s})$. Because some calculated currents had very slow rise times, we estimated the $10-90 \%$ rise time from the response to the longest $(12 \mathrm{~ms})$ pulses. We averaged the rise times and decay time constants from individual fitted mechanisms to compare with experimental data. Calculated responses were plotted and fitted in IGOR PRO (Wavemetrics).

Results are reported as the mean $\pm \mathrm{SD}$ of the mean.

\section{Results \\ Whole-cell recording of homomeric and heteromeric $\alpha \mathrm{K} 276 \mathrm{E}$ receptors}

The aim of the present study is to revisit our original finding that the K276E mutation impairs gating (Lewis et al., 1998), in the light of the recent evidence that Cys-loop receptors traverse one or more intermediate shut states during activation (Beato et al., 2004; Burzomato et al., 2004; Plested et al., 2007; Krashia et al., 2011).

Our initial characterization by whole-cell concentration-response curves showed that the mutation profoundly decreases glycine sensitivity in both homomeric (Fig. $1 A$ ) and heteromeric channels (data not shown). In heteromeric $\alpha 1 \mathrm{~K} 276 \mathrm{E} \beta$ channels, the $E C_{50}$ was $2.8 \mathrm{~mm}$ ( $n=5$ cells; two-unit likelihood interval, $1.8-4.5 \mathrm{~mm}$ ) and the Hill slope was 1.13 (two-unit likelihood interval, 0.94-1.39). The increase in $E C_{50}$ compared with wildtype receptors (Burzomato et al., 2003) is $\sim 30$-fold in heteromers, similar to that previously reported in our preliminary studies in Xenopus oocytes (Lewis et al., 1998). These macroscopic measurements were performed to help in the choice of the concentrations to be used for single-channel recording, but they are not as informative as the single-channel $P_{\text {open }}$ curve, which gives an estimate of the maximal activation on an absolute scale and is in principle unaffected by desensitization (Fig. 2B).

\section{Single-channel recordings of homomeric $\alpha 1 \mathrm{~K} 276 \mathrm{E}$ receptors} In cell-attached recordings from homomeric $\alpha 1 \mathrm{~K} 276 \mathrm{E}$ receptors, glycine (10-200 mM) evoked sparsely distributed openings (Fig. $1 C)$, whose apparent duration did not show much concentration dependence [mean open time $0.214 \pm 0.009 \mathrm{~ms}$ and $0.32 \pm 0.04$ $\mathrm{ms}$ at 10 and $200 \mathrm{~mm}$, respectively, $n=2$ patches; compare to 0.7 $\mathrm{ms}$ for wild-type homomeric receptors, even at the lowest concentration tested, $10 \mu \mathrm{M}$ glycine (Beato et al., 2004)]. We observed similar channel activity at 50 and $100 \mathrm{~mm}$ glycine (five patches in total) and in six other patches at 10 and $200 \mathrm{~mm}$, where activations were too few to be analyzed sensibly ( $<500$ activations in 5-10 $\mathrm{min}$ ).

Groups of closely spaced openings, typical of wild-type nicotinic and glycine receptor bursts, were not seen with mutant homomeric receptors, and activations appeared to consist mainly of single openings (Fig. $1 C$; mean number of apparent openings per burst, $1.08 \pm 0.02$ and $1.16 \pm 0.009 ; n=2$ patches at 10 and 200 $\mathrm{mm}$ glycine, respectively). The briefness of homomeric activations was confirmed by the fact that the deactivation of mutant macroscopic currents (Fig. $1 B$ ) is nearly fivefold faster than for wild-type receptors (time constant, $1.0 \pm 0.2 \mathrm{~ms}, n=7$, vs $4.7 \pm$ $0.5 \mathrm{~ms}$, respectively, $n=9$ patches).

\section{Single-channel recordings of the heteromeric $\alpha 1 \mathrm{~K} 276 \mathrm{E} \beta$ receptor}

Inclusion of the $\beta$ subunit is not necessary for a functional glycine receptor, but native synaptic receptors are heteromers of $\alpha$ and $\beta$ 
A

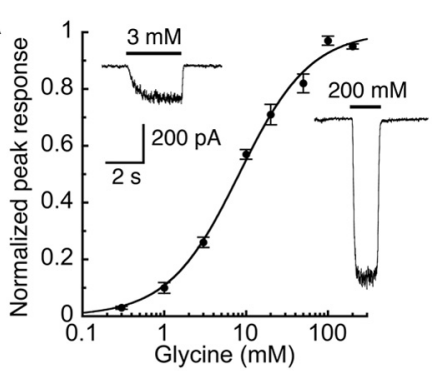

B $30 \mathrm{~mm}$ alycine

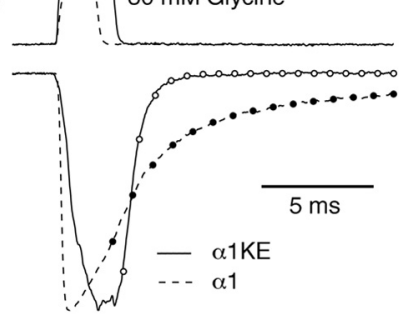

C
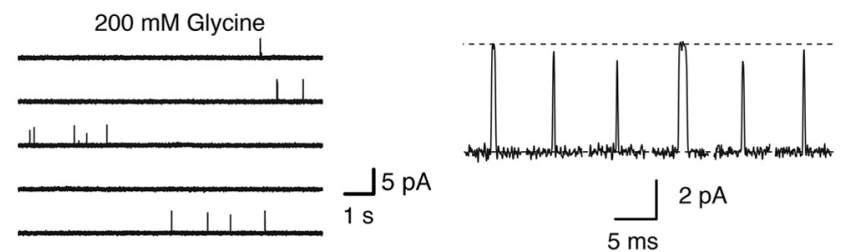

D
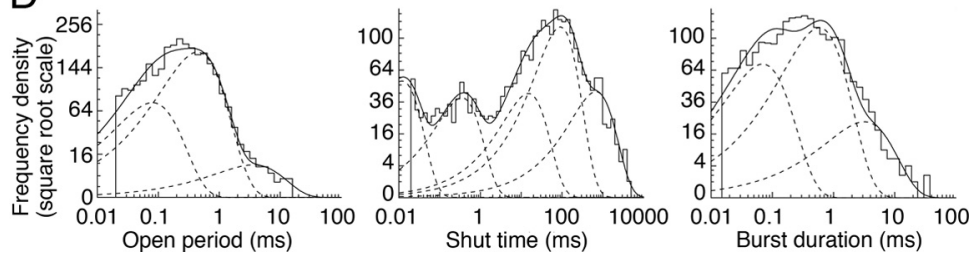

Figure 1. The properties of $\alpha 1 \mathrm{~K} 276 \mathrm{E}$ homomeric glycine receptors. $\boldsymbol{A}$, The concentration-response relation for $\alpha 1 \mathrm{~K} 276 \mathrm{E}$ homomeric glycine receptors. Points represent average data for $n=8$ cells. The line is the fit of the Hill equation to the data $\left(E C_{50}=8.2 \pm 0.9 \mathrm{~mm}\right.$, two-unit likelihood interval $6.4-9.4 \mathrm{~mm} ; n_{\mathrm{H}}=1.04 \pm 0.03$, two-unit likelihood interval $0.96-1.13)$. Sensitivity to glycine is greatly reduced compared with wild-type homomeric receptors, which have an $E C_{50}$ of $78.8 \mu \mathrm{m}$ (Beato et al., 2002). Insets show representative whole-cell currents. $\boldsymbol{B}$, Outside-out patch recordings of nonequilibrium responses of wild-type and $\alpha 1 \mathrm{~K} 276 \mathrm{E}$ homomeric receptors to $30 \mathrm{~mm}$ glycine jumps. The decay of wild-type responses was fitted with two exponential components (filled circles, $\tau_{1}=1.9 \mathrm{~ms}, 69 \%, \tau_{2}=13 \mathrm{~ms}$ ). The decay of the mutant responses was much faster and well fitted with a single component (open circles, $\tau=642 \mu \mathrm{s}$ ). $\boldsymbol{C}$, Equilibrium single-channel recordings of $\alpha 1 \mathrm{~K} 276 \mathrm{E}$ homomeric glycine receptors. Activations were sparse at a range of concentrations from 10 to $200 \mathrm{~mm}$ glycine, and no clustering was observed. Openings were brief, with mean amplitude of $5.1 \pm 0.2 \mathrm{pA}$ ( $n=10$ patches) at a nominal holding potential of $-80 \mathrm{mV}$. Many openings were shorter than the rise time of the filter, as shown also by the examples of individual activations at $200 \mathrm{~mm}$ glycine in the right panel. $\boldsymbol{D}$, Dwell-time distributions at $200 \mathrm{~mm}$ glycine. The open period distribution (left) was fitted with three components (dashed lines): $\tau_{1}=79 \mu \mathrm{s}(29 \%)$, $\tau_{2}=430 \mu \mathrm{s}(68 \%)$, and $\tau_{3}=3.5 \mathrm{~ms}(3 \%)$. The shut time distribution (middle) was fitted with a mixture of five exponential components: $\tau_{1}=12 \mu \mathrm{s}(18 \%), \tau_{2}=340 \mu \mathrm{s}(13 \%), \tau_{3}=15 \mathrm{~ms}(15 \%), \tau_{4}=89 \mathrm{~ms}(39 \%)$, and $\tau_{5}=666$ ms (15\%). In general, few short shuttings were detected, and the distributions of open and shut periods exhibited little concentration dependence. To plot burst durations (right) and count the number of openings per burst, the record was divided into bursts by using a critical shut time duration of $3 \mathrm{~ms}$. The burst duration distribution was fitted with a mixture of three exponential densities $\left[\tau_{1}=70 \mu \mathrm{s}(34 \%), \tau_{2}=590 \mu \mathrm{s}(55 \%)\right.$, and $\left.\tau_{3}=3.2 \mathrm{~ms}(11 \%)\right]$.

subunits. As seen clearly from Figure 2, the K276E mutant heteromeric receptor opened far more frequently than its homomeric counterpart. Furthermore, at concentrations $>300 \mu \mathrm{M}$, activations appeared in clusters separated by long, presumably desensitized, shut intervals, as we had shown for the wild-type $\alpha 1 \beta$ glycine receptor. The mean amplitude of single-channel currents was $3.1 \pm 0.1 \mathrm{pA}(n=17$ patches), indistinguishable from wild-type receptor (Burzomato et al., 2004).

The apparent open and shut period distributions from idealized single-channel records are depicted in Figure $2 B$. The distributions were fitted empirically with mixtures of exponential probability density functions and the fitting results are summarized in Tables 1 and 2 .

Three components were necessary to fit well the open period distributions at all concentrations. The values of the two slower time constants remained relatively stable across concentrations, whereas the briefest openings were somewhat shorter in $300 \mu \mathrm{M}$ records (time constant, $64 \mu \mathrm{s}$ ) than at clustering concentrations (time constant, $>100 \mu \mathrm{s})$. One possible explanation for the change of the shortest component is that, at the lowest concentration, that component represents openings from partially liganded receptors that become too rare to be detectable at higher concentrations (as supported by subsequent analysis). An important observation was that, even at the highest concentrations, open period distributions retained at least three exponential components, in contrast to wild-type receptors, for which the equivalent distribution appears to be monoexponential (Burzomato et al., 2004). This observation suggests that in the mutant there is more than one fully liganded open state.

Distributions of apparent shut times were fitted well with a mixture of at least four exponential probability density functions. There were consistently two fast shut time components (mean time constants, $29 \pm 5 \mu \mathrm{s}$ and $310 \pm 100 \mu \mathrm{s}$ at $300 \mu \mathrm{M}$ glycine, $n=4$ patches) that remained very similar at all concentrations. The time constants of the two slowest components (45 and $242 \mathrm{~ms}$, $300 \mu \mathrm{M}$ glycine) were greatly reduced at high concentrations ( 1 and $4 \mathrm{~ms}$ at 100 mm glycine). The number of long desensitized shut intervals was low at all concentrations, so the fits of those intervals with a separate exponential component were not robust. Nevertheless, these intercluster intervals were about 300 times longer than the next slower (intracluster) shut time component, so there was no ambiguity in dividing records into clusters using an appropriate critical time interval, a key step in the subsequent analysis.

\section{Single-channel $\boldsymbol{P}_{\text {open }}$ curve}

The traces in Figure $3 A$ show the increase in $P_{\text {open }}$ with glycine concentration for $\alpha 1 \mathrm{~K} 276 \mathrm{E} \beta$ receptors. This is strikingly different from that of wild-type receptors (Fig. 3B, dashed curve, data from Burzomato et al., 2004). First, the maximum open probability during a cluster was $45 \%$ (two-unit likelihood interval $0.39-0.51$ ), less than half of that seen for wild type. Second, the concentration of glycine that elicited half-maximal activation was $6.2 \mathrm{~mm}$ (two-unit likelihood interval, $4.4-8.5 \mathrm{~mm}$ ). This is 100 fold greater than for wild type and 20-fold higher than for the extracellular domain mutant $\alpha 1 \mathrm{~A} 52 \mathrm{~S}$ (Plested et al., 2007). The Hill slope is somewhat poorly defined (1.6; two-unit likelihood interval, 0.952.5 ), probably because of the small number (four) of points on the curve. Because we deliberately worked under conditions of low receptor expression (see Materials and Methods), few recordings contained channel openings, making it difficult to collect data at the lowest concentrations where clusters were seen.

\section{Fitting mechanisms to single-channel data: introduction to fitting}

We investigated the activation mechanism of the $\alpha 1 \mathrm{~K} 276 \mathrm{E} \beta$ heteromeric glycine receptor by fitting $>40$ postulated mechanisms 
A

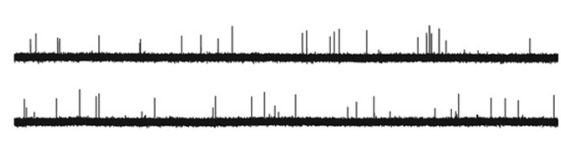

$300 \mu \mathrm{M}$

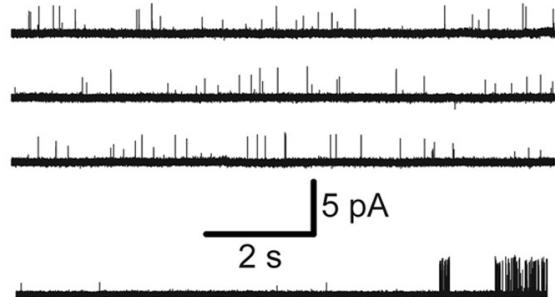

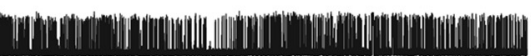

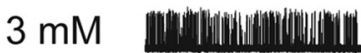
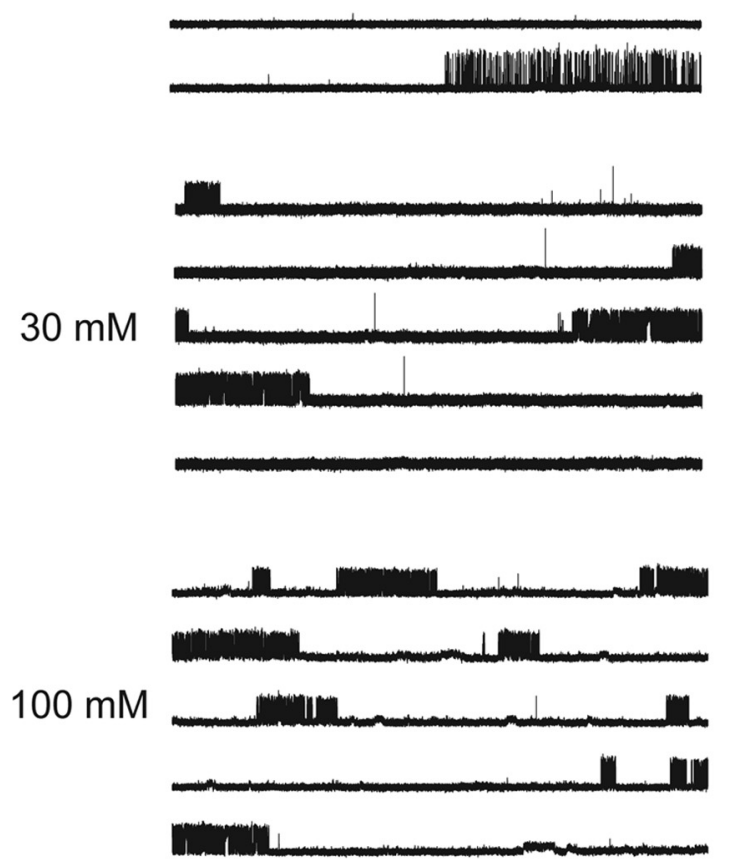
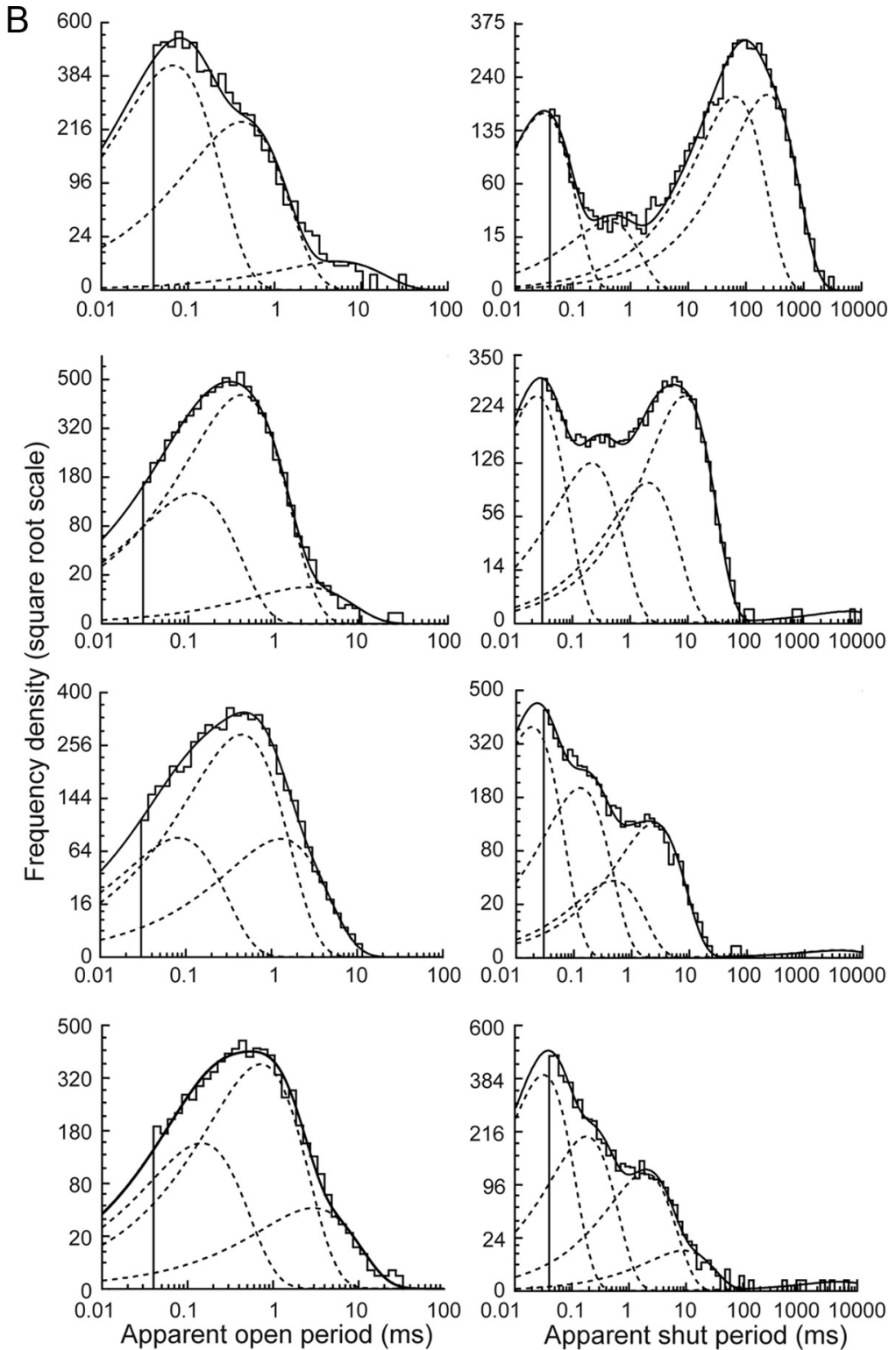

Figure 2. Empirical fits to single-channel recordings of $\alpha 1 \mathrm{~K} 276 \mathrm{E} \beta$ heteromeric glycine receptors. A, Continuous runs of 50 s of representative $\alpha 1 \mathrm{~K} 276 \mathrm{E} \beta$ heteromeric glycine receptor activity at several glycine concentrations. At the lowest concentration where activations were observed ( $300 \mu \mathrm{m}$ glycine), most events were isolated single openings. At concentrations of glycine of $3 \mathrm{~mm}$ and above, activations were grouped into clusters separated by long (1-100 s) quiescent periods. The clusters were shorter at higher concentrations. Data were filtered at 3-5 kHz. A discharge of the amplifier feedback capacitor can be seen in the intercluster gaps in each of the first four traces for the $30 \mathrm{~mm}$ patch. In the $100 \mathrm{~mm}$ patch shown here, the very low baseline noise level (about $150 \mathrm{fA}$ at $5 \mathrm{kHz}$ ) revealed sporadic activations of a slow channel, presumably endogenous to $\mathrm{HEK} 293$ cells, with a conductance of $\sim 500 \mathrm{fS}$, which was occasionally seen in other records. $\boldsymbol{B}$, Distributions of idealized single-channel open and shut periods were fitted with mixed exponential densities. Components are shown with dotted lines (see Tables 1 and 2 for average values). Three components were used for open times at every concentration. Five components were used for shut times. At the lowest concentration of glycine $(300 \mu \mathrm{M})$, two components described the shut times that divided bursts (that is, those longer than the critical time, $0.6 \mathrm{~ms})$. At all other concentrations, four components described the majority of shuttings within clusters, and the fifth component loosely fitted the long desensitized sojourns between clusters. Note that, with increasing concentration, apparent open times lengthen and apparent shut times shorten. However, unlike wild-type receptors, the longer intracluster shut times remain, even at the highest concentration (100 mм glycine).

Table 1. Empirical fit of mixtures of exponential densities to the distributions of apparent open times from $\alpha 1 \mathrm{~K} 276 \mathrm{E} \beta$ receptors

\begin{tabular}{llclcl}
\hline Gly $(\mu \mathrm{m})$ & $n$ & $\tau 1$ (ms) (area [\%]) & $\tau 2$ (ms) (area [\%]) & $\tau 3$ (ms) (area [\%]) & Mean open time (ms) \\
\hline 300 & 4 & $0.064 \pm 0.008(57 \pm 4)$ & $0.36 \pm 0.04(39 \pm 2)$ & $3 \pm 1(4 \pm 2)$ & $0.24 \pm 0.01$ \\
3000 & 2 & $0.108 \pm 0.005(25 \pm 1)$ & $0.49 \pm 0.07(72 \pm 2)$ & $2.2 \pm 0.1(3 \pm 1)$ & $0.44 \pm 0.06$ \\
10,000 & 4 & $0.12 \pm 0.03(31 \pm 3)$ & $0.52 \pm 0.10(65 \pm 2)$ & $2.4 \pm 0.6(4 \pm 1)$ & $0.46 \pm 0.08$ \\
30,000 & 3 & $0.11 \pm 0.04(31 \pm 13)$ & $0.67 \pm 0.09(62 \pm 11)$ & $3 \pm 1(7 \pm 4)$ & $0.61 \pm 0.03$ \\
100,000 & 4 & $0.20 \pm 0.05(42 \pm 13)$ & $0.93 \pm 0.15(52 \pm 10)$ & $4 \pm 1(6 \pm 4)$ & $0.72 \pm 0.04$ \\
\hline
\end{tabular}

Time constants and areas are expressed as mean \pm SD of the mean. The mean apparent open time values are obtained directly from the idealized records (after resolution is imposed) as the average of all apparent open periods. 
Table 2. Empirical fit of mixtures of exponential densities to the distributions of apparent shut times from $\alpha 1 \mathrm{~K} 276 \mathrm{E} \beta$ receptors

\begin{tabular}{llllccc}
\hline Gly $(\mu \mathrm{m})$ & $n$ & $\tau 1(\mathrm{~ms})$ (area [\%]) & $\tau 2$ (ms) (area [\%]) & $\tau 3$ (ms) (area [\%]) & $\tau 4$ (ms) (area [\%]) & Intercluster (s) (area [\%]) \\
300 & 4 & $0.029 \pm 0.005(26 \pm 2)$ & $0.31 \pm 0.10(6 \pm 1)$ & $45 \pm 14(38 \pm 13)$ & $242 \pm 21(30 \pm 13)$ & \\
3000 & 2 & $0.023 \pm 0.001(37 \pm 3)$ & $0.18 \pm 0.04(17.6 \pm 0.3)$ & $1.4 \pm 0.6(12.9 \pm 0.4)$ & $7 \pm 1(32 \pm 2)$ & $4 \pm 2(0.05 \pm 0.05)$ \\
10,000 & 4 & $0.042 \pm 0.015(43 \pm 4)$ & $0.17 \pm 0.06(21 \pm 4)$ & $0.9 \pm 0.3(23 \pm 4)$ & $4 \pm 1(13 \pm 5)$ & $3 \pm 1(0.05 \pm 0.03)$ \\
30,000 & 3 & $0.017 \pm 0.002(44 \pm 5)$ & $0.09 \pm 0.02(22 \pm 5)$ & $0.30 \pm 0.09(16 \pm 5)$ & $2.2 \pm 0.2(17.5 \pm 0.3)$ & $2 \pm 1(0.03 \pm 0.03)$ \\
100,000 & 4 & $0.025 \pm 0.004(45 \pm 5)$ & $0.12 \pm 0.03(27 \pm 1)$ & $1.0 \pm 0.4(16 \pm 2)$ & $4.2 \pm 0.7(12 \pm 3)$ & $4 \pm 2(0.15 \pm 0.03)$ \\
\hline
\end{tabular}

Time constants and areas are expressed as mean \pm SD of the mean.

A

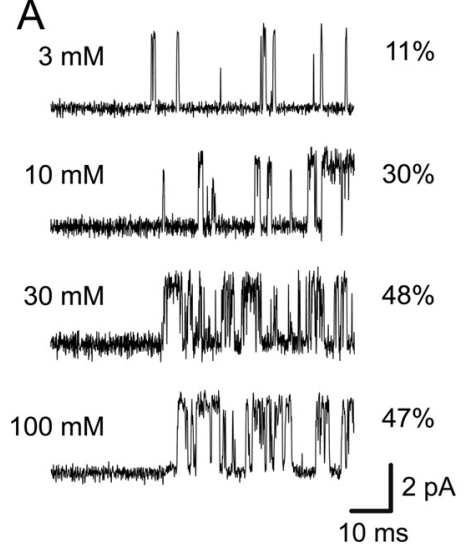

B

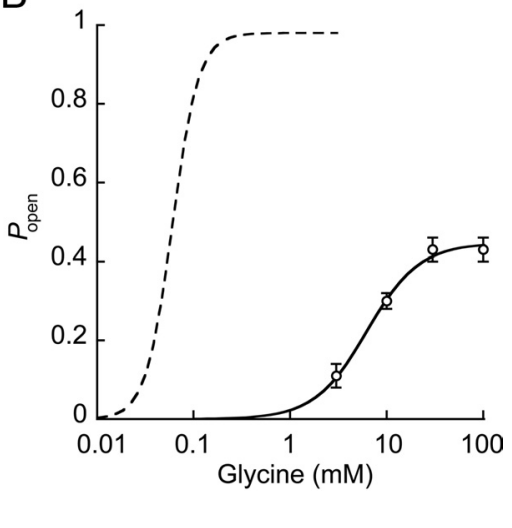

Figure 3. The probability of a channel being open during clusters of the $\alpha 1 \mathrm{~K} 276 \mathrm{E} \beta$ heteromeric glycine receptor activations. $\boldsymbol{A}$, Representative activations from the initial segment of a cluster for the range of concentrations used to construct the $P_{\text {open }}$ curve. The open probability for the cluster is indicated in the right column. At higher concentrations, longer groups of openings appear but a complex mixture of shut times (up to about $10 \mathrm{~ms}$ ) are present at all concentrations. $\boldsymbol{B}$, Cluster open probability was plotted against glycine concentration (open circles; $2-4$ patches, $11-73$ clusters per point). The maximum open probability obtained from the fitted Hill equation (solid line) was $45 \pm 3 \%$, much less than for wild-type homomeric or heteromeric glycine receptors containing the $\alpha 1$ subunit. The $E C_{50}$ was $6.2 \pm 0.9 \mathrm{~mm}$, and Hill slope was $1.6 \pm 0.4$. Wild-type heteromeric receptor open probability [dashed line, shown here for comparison, as calculated in Burzomato et al. (2004)] reached a maximum of $97 \%$, with $E C_{50}$ of $0.06 \mathrm{~mm}$ and Hill slope of 3.4 .

to single-channel data using the HJCFIT method (including variants of the del Castillo-Katz scheme). Here we present the results of fitting four different kinetic mechanisms: two that describe wild-type glycine receptors well, and the two mechanisms that best fitted the mutant.

In this analysis, mechanisms are fitted directly to the singlechannel data (appropriately divided in bursts and clusters; see Colquhoun et al., 2003, and Sivilotti, 2010, for more details of the procedure), and the fit is repeated on independent sets of patches (three in the present study). Each set contains four recordings obtained at different glycine concentrations, spanning the whole of the concentration-open probability curve.

The second step in the analysis is to compare the models and test the quality of the fits. This is done by using each model and the rate constant values obtained in the fits to calculate predictions of the following experimental single-channel observations: apparent open and shut time distributions, open and shut time correlations, and $P_{\text {open }}-$ concentration relations. In addition, we calculated macroscopic current responses to pulses of glycine and the activation and deactivation time constants. These model predictions were then compared with the experimental data from single-channel and fast concentration jump experiments.

We will take the "flip" scheme and the rate constants reported in Burzomato et al. (2004) as our reference for the activation mechanism of wild-type glycine channels.

\section{Scheme 1: "Jones-Westbrook" mechanism}

We started with a mechanism (Fig. 4, Scheme 1) widely used in studies of ligand-gated receptors, and originally proposed for the

$\mathrm{GABA}_{\mathrm{A}}$ receptor by Jones and Westbrook (1995). Though we did not favor this mechanism in our earlier studies, it fits well data from heteromeric or homomeric wild-type glycine receptors (Burzomato et al., 2004). In the present case, in contrast, it is clear that the Jones-Westbrook mechanism does not fit the mutant receptor data.

Examples of $P_{\text {open }}$ curve, open-shut time distributions, and correlation plots for one of the sets fitted with Scheme 1 are depicted in Figure 4, $B$ and $C$. The results of the fits to all three datasets are summarized in Table 3. The description of the experimental data by these fits was unsatisfactory, mainly because this model has a single fully liganded open state. As a consequence, it cannot predict open-shut time correlations at the highest glycine concentration. The model is poor also at describing the apparent open period distributions. Finally, the variability of fitted rate constants between sets was high ( $\mathrm{Ta}$ ble 3). Unsurprisingly, given this variability, the shape of the $P_{\text {open }}$-concentration response predicted by the fits of this scheme was also inconsistent. Two of the three sets predicted biphasic $P_{\text {open }}-$ concentration response relations, with a peak at about $50 \mathrm{~mm}$ (Fig. $4 B$, continuous curve). The fit to the third set predicted a monotonically growing $P_{\text {open }}$ curve with two very distinct components (data not shown). These fits also predicted responses to concentration jumps unlike those measured in outside-out patches (see Concentration jumps: a comparison between experimental responses and those predicted by simulations, below; see Fig. 10).

\section{Scheme 2: flip mechanism}

The mechanism depicted in Figure 5A, Scheme 2, is the flip mechanism, as proposed by Burzomato et al. (2004) for heteromeric and homomeric wild-type glycine receptors and subsequently used also to describe activations of the muscle nicotinic receptor (Lape et al., 2008, 2009). This mechanism described well also the activation of glycinergic $\alpha 1 \mathrm{~A} 52 \mathrm{~S} \beta$ mutant channels (Plested et al., 2007) and wild-type $\alpha 2$ homomers (Krashia et al., 2011). This scheme introduces short-lived intermediate shut states between the resting and the open conformations, and explains the strong apparent interaction between binding sites in wild-type receptors as nothing more than simple equilibrium between low- and highaffinity shut states of independent binding sites.

An example of a fit of Scheme 2 is shown in Figure 5, $B$ and $C$ (the same dataset as in Fig. 4). All fits with Scheme 2 (also with Schemes 3 and 4) were done with the assumption of independent binding sites. This assumption was imposed by the following constraints: $k_{+1}=k_{+2}=k_{+3}$ and $k_{-1}=k_{-2}=k_{-3}$. 
A

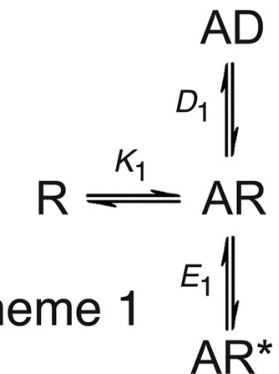

C

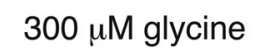

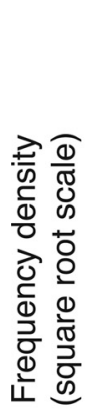
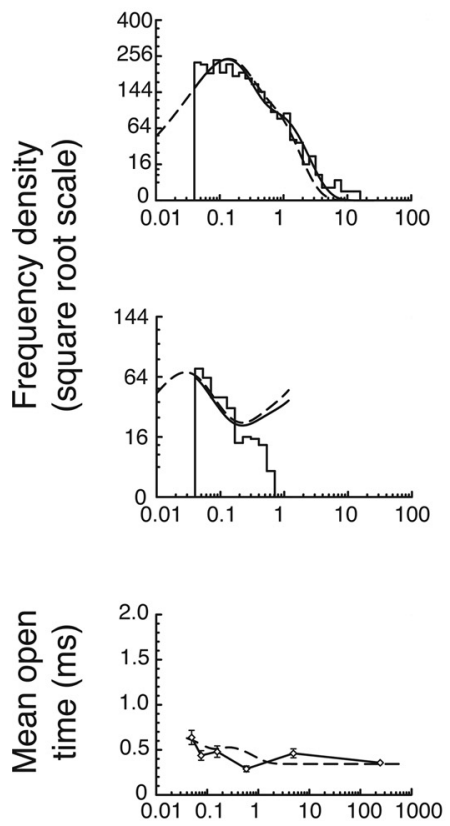

$A_{2} D$<smiles>C1C2C3C1C1C2C31</smiles><smiles>C1CC1</smiles>

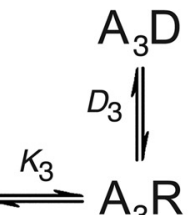

$A_{2} R \rightleftharpoons A_{3} R$

$E_{2} \|$

$A_{2} R^{*}$

$E_{3} \|$

$A_{3} R^{*}$
B

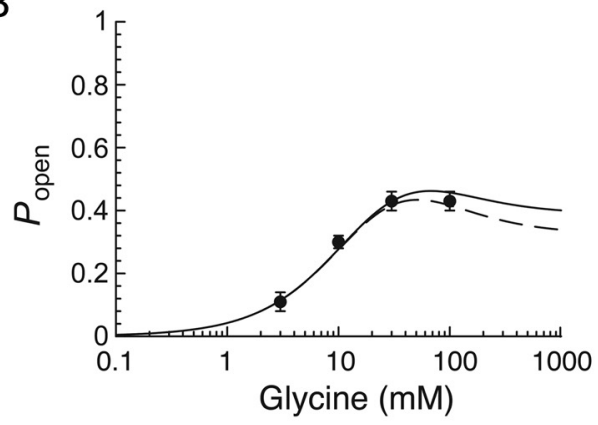

$10 \mathrm{mM}$ glycine
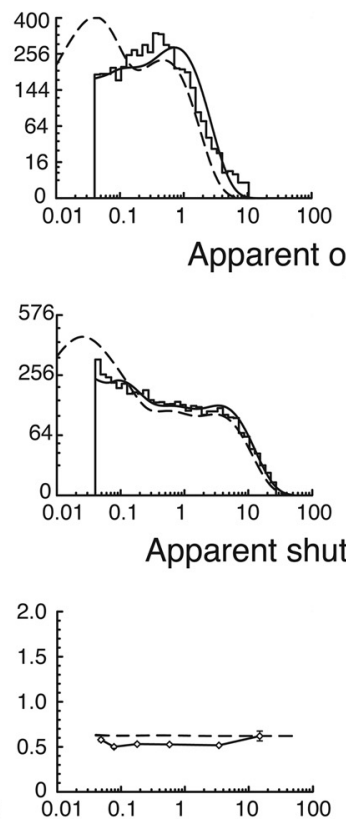

Mean apparent shut time (ms)

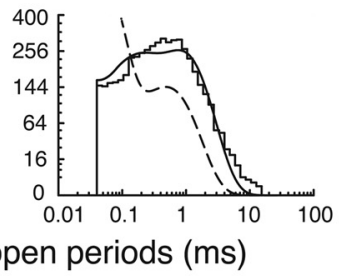

$100 \mathrm{mM}$ glycine

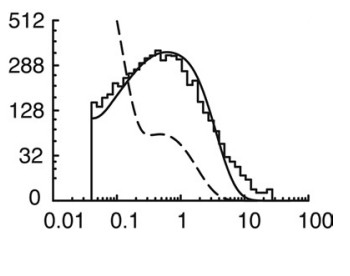

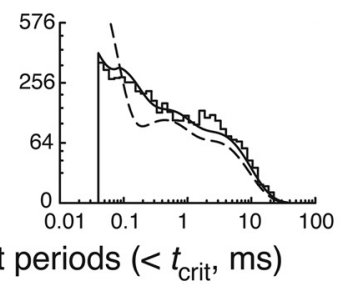
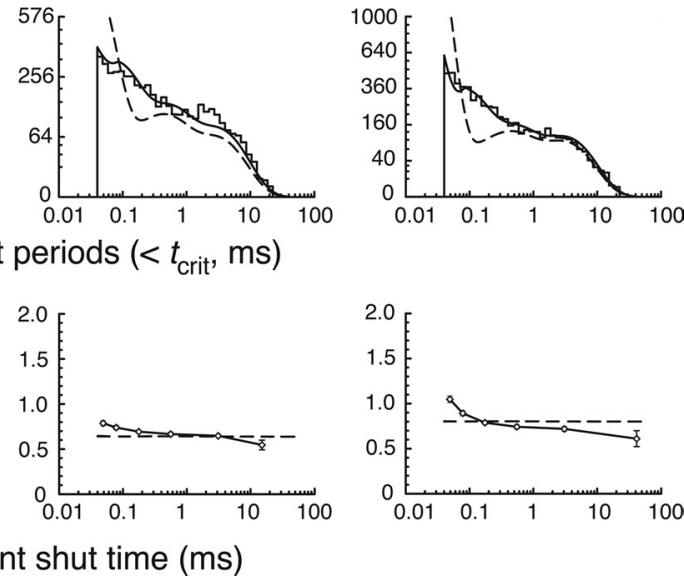

Figure 4. Fits of a mechanism with distal shut states to the $\alpha 1 \mathrm{~K} 276 \mathrm{E} \beta$ heteromeric receptor single-channel data. $A$, Scheme 1 represents a receptor with three binding sites and three open states (indicated by asterisks) directly connected to the monoliganded, diliganded, and fully liganded resting shut states. Directly connected to the resting bound shut states are short-lived shut states. Fits with this mechanism were done assuming that the binding sites were not independent. Thus, all rate constants in these fits were free parameters (18 in total). $\boldsymbol{B}$, The solid line is the $P_{\text {open }}$ curve calculated from the values of the rate constants fitted to this set and taking missed events into account. Data points are cluster open probabilities as in Figure $3 B$. The dashed line is the predicted $P$ open curve at ideal resolution. $C$, The results of Scheme 1 fit to a dataset containing four single-channel records. The histograms are the experimental open (top row) and shut (middle row) period distributions. The continuous curves are the distributions predicted from the fitted rate constants (taking missed events into account). The dashed lines are calculated from the same rate constant values and are the predicted distributions expected for a perfect experimental resolution [no missed events; Eqs. 3.64 and 3.90 in Colquhoun and Hawkes (1982), for open and shut times, respectively]. The bottom row plots show the open-shut time correlations in the records of this set (circles with error bars and connecting lines) and the predictions for these correlations (dashed lines) calculated from the fitted rate constants.

The quality of predictions with Scheme 2 was comparable to those with Scheme 1, despite fewer free parameters (14 compared with 18 in Scheme 1). Predicted $E C_{50}$, maximum $P_{\text {open }}$, and Hill slope ( $9.2 \mathrm{~mm}, 0.49$, and 1.3 , respectively) were fairly close to those estimated by direct fit of Hill equation to the experimental points (Fig. 3), although the predicted maximum open probability was a little higher than observed (Fig. 5B).

The fits of Scheme 2 predicted reasonably well the shape of shut time distributions. However, the predicted shapes of open period distributions were poor at all concentrations. This is simply because, at saturating glycine concentrations, Scheme 2 has only one open state and therefore predicts a single exponential distribution of open times. But at the highest glycine concentration (100 mM) tested, the distribution of apparent open times was observed to have at least three components. This is the same effect that was visible in fits of Scheme 1 (which also has only one open state for the saturated receptor). Since the experimental singlechannel $P_{\text {open }}$ curve is relatively flat from $10 \mathrm{~mm}$ upward, it seems likely that $100 \mathrm{~mm}$ glycine is close to being a saturating concentration, and this implies that a mechanism is needed that has more than one fully bound open state.

Scheme 2 also failed to predict the open-shut time correlation at saturating agonist concentrations (Fig. 5C, compare prediction shown as dashed line and the data points). This is because it has only one fully liganded open state and, when there is one open state, no correlations are predicted (Colquhoun and Hawkes, 1987).

The average rate constants estimated with Scheme 2 are given in the first column of Table 4. We have to be cautious in interpreting them, because the scheme gave a relatively poor description of the experimental data. The values for the fully liganded opening step appeared to be quite consistent across sets, and the 
Table 3. Estimated parameters from Scheme 1 fitted to $\alpha 1 \mathrm{~K} 276 \mathrm{E} \beta$ glycine receptor single-channel data

\begin{tabular}{|c|c|}
\hline & Scheme 1: Jones-Westbrook \\
\hline$\alpha_{1}\left(\mathrm{~s}^{-1}\right)$ & $11,400 \pm 20 \%$ \\
\hline$\beta_{1}\left(\mathrm{~s}^{-1}\right)$ & $1160 \pm 48 \%$ \\
\hline$\alpha_{2}\left(s^{-1}\right)$ & $9260 \pm 61 \%$ \\
\hline$\beta_{2}\left(s^{-1}\right)$ & $49,400 \pm 55 \%$ \\
\hline$\alpha_{3}\left(s^{-1}\right)$ & $24,700 \pm 53 \%$ \\
\hline$\beta_{3}\left(s^{-1}\right)$ & $57,400 \pm 47 \%$ \\
\hline$d_{-1}\left(s^{-1}\right)$ & $291 \pm 52 \%$ \\
\hline$d_{+1}\left(s^{-1}\right)$ & $5090 \pm 53 \%$ \\
\hline$d_{-2}\left(s^{-1}\right)$ & $107,000 \pm 98 \%$ \\
\hline$d_{+2}\left(s^{-1}\right)$ & $5200 \pm 14 \%$ \\
\hline$d_{-3}\left(s^{-1}\right)$ & $1330 \pm 69 \%$ \\
\hline$d_{+3}\left(s^{-1}\right)$ & $4000 \pm 54 \%$ \\
\hline$k_{-1}\left(s^{-1}\right)$ & $6550 \pm 12 \%$ \\
\hline$k_{+1}\left(\mathrm{M}^{-1} \mathrm{~s}^{-1}\right)$ & $2.06 \times 10^{6} \pm 60 \%$ \\
\hline$k_{-2}\left(s^{-1}\right)$ & $15,200 \pm 27 \%$ \\
\hline$k_{+2}\left(M^{-1} s^{-1}\right)$ & $1.76 \times 10^{6} \pm 25 \%$ \\
\hline$k_{-3}\left(s^{-1}\right)$ & $231,000 \pm 93 \%$ \\
\hline$k_{+3}\left(M^{-1} s^{-1}\right)$ & $5.54 \times 10^{6} \pm 95 \%$ \\
\hline$E_{1}=\beta_{1} / \alpha_{1}$ & $0.120 \pm 54 \%$ \\
\hline$E_{2}=\beta_{2} / \alpha_{2}$ & $5.98 \pm 11 \%$ \\
\hline$E_{3}=\beta_{3} / \alpha_{3}$ & $4.23 \pm 43 \%$ \\
\hline$D_{1}=d_{+1} / d_{-1}$ & $11.6 \pm 50 \%$ \\
\hline$D_{2}=d_{+2} / d_{-2}$ & $6.13 \pm 84 \%$ \\
\hline$D_{3}=d_{+3} / d_{-3}$ & $3.94 \pm 25 \%$ \\
\hline$K_{1}=k_{-1} / k_{+1}(\mu \mathrm{m})$ & $6310 \pm 46 \%$ \\
\hline$K_{2}=k_{-2} / k_{+2}(\mu \mathrm{m})$ & $9580 \pm 32 \%$ \\
\hline$K_{3}=k_{-3} / k_{+3}(\mu \mathrm{m})$ & $4330 \pm 27 \%$ \\
\hline
\end{tabular}

Rate constants are means of fits to three data sets \pm the coefficient of variation of the mean. Equilibrium constants $(E, D$, and $K)$ are the mean values of the three values calculated for each separate data set. Scheme 1 has 18 free parameters.

opening rate remained almost as high as in wild type $\left(86,000 \mathrm{~s}^{-1}\right.$ compared to $129,000 \mathrm{~s}^{-1}$ for wild type), whereas flipping appeared to be considerably impaired by the mutation (the fully liganded flipping equilibrium constant was 0.32 , about 100 -fold less than in wild type; Burzomato et al., 2004).

\section{Scheme 3: primed mechanism}

The existence of more than one open time component at saturating glycine concentrations suggested that it might be necessary to use a primed mechanism. This is a general mechanism (Mukhtasimova et al., 2009) in which each subunit can flip independently, rather than in a concerted fashion as in the flip mechanism. The flip mechanism (Fig. 5A) can be regarded as a subset of the primed model, if $F^{\prime}$ in the primed model (Fig. 6A) is taken as equivalent to the flipped state and all the rows below this (those with $F^{\prime \prime}$ and $F^{\prime \prime \prime}$ ) are deleted.

Although this mechanism has considerable appeal from physical and symmetry arguments, technical problems arise because it has many more states (both shut and open) than flip, and consequently more free parameters. In our previous work, 18 free parameters were well estimated from wild-type heteromeric glycine receptor equilibrium single-channel recordings (Burzomato et al., 2004). However, the open and shut time distributions of the mutant $\alpha 1 \mathrm{~K} 276 \mathrm{E} \beta$ are more complex than those of the wild-type receptor, perhaps consistent with greater information content within the records. This observation encouraged us to try to fit a subset of the primed mechanism to single-channel data. Scheme 3 (Fig. 6A) shows a subset of the full primed mechanism, where (as in flip, Scheme 2) the unliganded branch is omitted on the grounds that, because it is so rarely visited, the data contain little or no information about it. Even this reduced version has 27 free parameters, almost twice as many as the flip model.

The results of the primed mechanism fits are summarized in the second column of Table 4 and an example of the fit to the same dataset as with previous schemes is shown in Figure 6. In this case, prediction of the apparent open period distribution is very accurate throughout the entire concentration range (Fig. $6 C$, top row) because the mechanism has multiple fully liganded open states. Distributions of apparent shut times and the negative correlation between open and shut time duration at high glycine (Fig. 6C, middle and bottom rows) are also predicted very well. The calculated $P_{\text {open }}$ curve overlapped well with the experimental points, and predicted $E C_{50}$, max $P_{\text {open }}$, and a Hill slope at the midpoint similar to the values obtained from the Hill equation fits to the data ( $6.3 \mathrm{~mm}, 0.42$, and 1.4 , respectively).

Although the fit is excellent, it was not possible to get good estimates of all 27 free parameters. The best-estimated rate constants were between fully liganded states (coefficient of variation smaller than $33 \%$, except for $\gamma_{3}^{\prime \prime}$ and $\delta_{3}^{\prime \prime}$, the rate constants for unpriming and priming of the second subunit in the transition between $A_{3} F^{\prime}$ and $A_{3} F^{\prime \prime}$ ) (Table 4; Fig. 6).

The maximum probability of a channel being open, $P_{\max }$, occurs when the binding sites are saturated (all triply liganded). As with other mechanisms, we can write the maximum $P_{\text {open }}$ in terms of an "effective efficacy," $E_{\text {eff: }}$ :

$$
P_{\max }=\frac{E_{\text {eff }}}{1+E_{\text {eff }}}
$$

where we now define the effective efficacy as

$$
E_{\mathrm{eff}}=\frac{E_{3}^{\prime} F_{3}^{\prime}+E_{3}^{\prime \prime} F_{3}^{\prime} F_{3}^{\prime \prime}+E_{3}^{\prime \prime \prime} F_{3}^{\prime} F_{3}^{\prime \prime} F_{3}^{\prime \prime \prime}}{1+F_{3}^{\prime}+F_{3}^{\prime} F_{3}^{\prime \prime}+F_{3}^{\prime} F_{3}^{\prime \prime} F_{3}^{\prime \prime \prime}}
$$

Note that once the channel is doubly and triply primed, it opens fairly effectively ( $E_{3}^{\prime \prime}$ and $E_{3}^{\prime \prime \prime}$ are 7 and 4 , respectively). It is the impairment of the "priming" steps, rather than the opening steps, that is responsible for the low effective efficacy when the channel is fully liganded $\left(E_{\text {eff }}=0.7\right)$ and, thus, for the low maximum open probability in the mutant receptor.

The forward rate constant of the triply primed fully liganded opening is $119,000 \mathrm{~s}^{-1}$. To the extent that rate constant values from different mechanisms can be compared, we note that this value is almost the same as the opening rate constant for fully liganded opening in wild-type heteromeric receptors $\left(129,000 \mathrm{~s}^{-1}\right.$, flip mechanism, Burzomato et al., 2004), but the mutant channel closes much faster $\left(27,600 \mathrm{~s}^{-1}\right)$. Glycine binding affinity was reduced in the resting conformation, compared with the wild-type heteromeric receptor, by a factor of about 6 .

\section{A reduced version of Scheme 3: elimination of rarely visited states}

The full primed model for three binding sites, omitting unliganded openings, has 106 rate constants. If all cycles obey microscopic reversibility, and the binding/unbinding rates are the same for any given conformation, the number of free rate constants to be estimated would still be 52 (compared with 14 free parameters for the flip mechanism; Fig. 5) (Colquhoun et al., 2004). It is beyond the capability of even single-channel measurements to estimate 52 free parameters from the data. One reason for this is that some states in the full mechanism will be visited rarely, so the data contain little information about the transition rates into and out of such states. It is therefore necessary to fit a subset of the full 
A<smiles>CP</smiles>

B

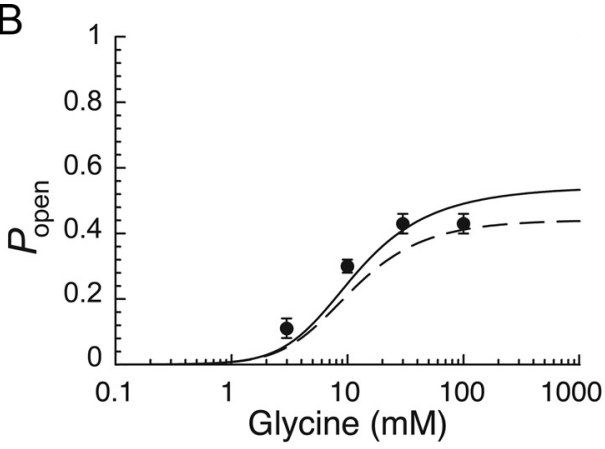

$30 \mathrm{mM}$ glycine
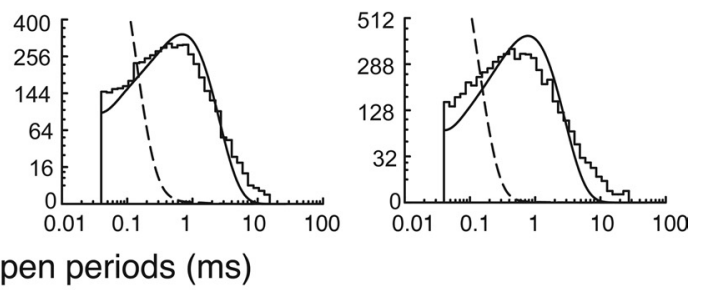

Apparent open periods (ms)
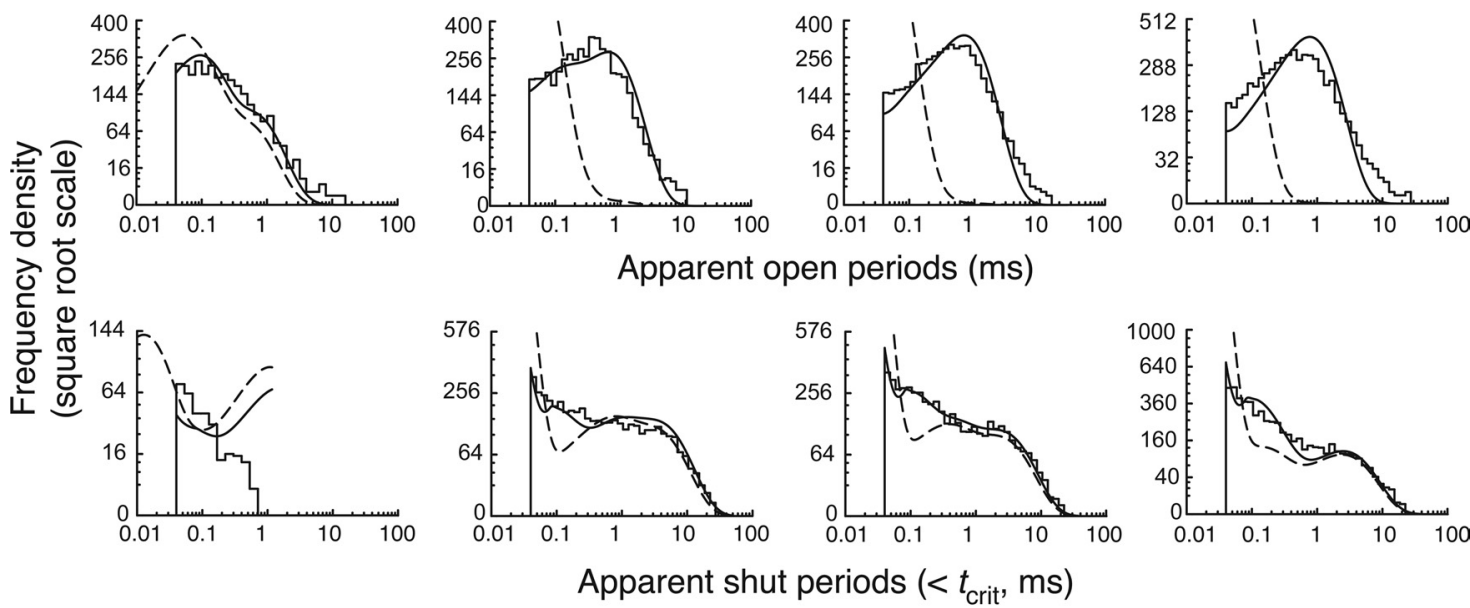

Apparent shut periods $\left(<t_{\text {crit }}\right.$, ms)
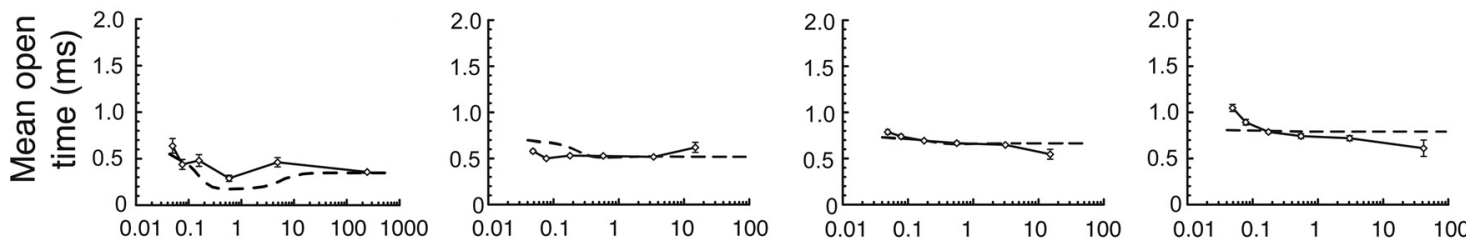

Mean apparent shut time (ms)

Figure 5. Fits of a flip mechanism to the heteromeric mutant receptor single-channel data. $\boldsymbol{A}$, Scheme 2 is a flip mechanism with three binding sites and three open states (indicated by asterisks), which can be accessed from resting liganded states through extra shut ("flipped") states. After imposing the constraints of binding site independence and microscopic reversibility, this mechanism had 14 free parameters. Average rate constants are given in Table 4. B, C, The results of Scheme 2 fitted to the same dataset as in Figure 4. For the details, see Figure 4. Note that open period distributions were not predicted well with the flip mechanism.

primed model from which rarely visited states have been deleted. Scheme 3 omits all states where fewer subunits are bound than primed. It is likely that the rarely visited states will not be the same ones in a mutant receptor as in the wild-type receptor. For example, states with less than full occupancy of the binding sites seem to be visited more often in the wild-type $\alpha 1 \beta$ receptor than they are in the $\alpha \mathrm{K} 276 \mathrm{E} \beta$ receptor. It makes sense, therefore, that different subsets of the full mechanism may need to be fitted to different receptor types. This does not preclude numerical comparisons of the values of rate constants that occur in both subsets.

Clearly Scheme 3, although already a subset of the full primed model, has too many parameters for all of them to be estimated with acceptable accuracy. It is quite possible to fit a mechanism in which parameters are not all well defined, and if a good fit is obtained, then one can say that the mechanism is compatible with the data. But this overparameterization is not to be recommended, except as a last resort, because it may cause problems in the fitting, such as multiple peaks in the likelihood surface, and consequently a risk that the fit will converge on a local submaximum and so return bad estimates even for the well defined pa- rameters. In general, we expect that more reliable estimates will be found if rarely visited states are removed from the mechanism altogether in such a way that all the remaining rate constants are reasonably well defined. This is how we arrived at a "reduced primed" mechanism.

We proceeded by looking at the equilibrium occupancies of the states in Scheme 3 and at the frequency with which the different states are visited. Low occupancy alone is not a sufficient criterion by which to remove states because, while very shortlived states may have a low occupancy, they nevertheless may be visited so frequently that their entry and exit rates are well defined by the data. There is a danger in this procedure, in that our choice is itself based on the results of the fit of an overparameterized scheme, and frequency and occupancy may not be accurately estimated. It is the best we can do and, to the best of our knowledge, the optimum way to simplify a model has not been investigated in a statistically rigorous way.

The occupancy of lower-liganded states in Scheme 3 was predicted to be very low, even at the lowest concentrations tested. The occupancy of the open states $A F^{\prime \star}, A_{2} F^{\prime \star}$, and $A_{2} F^{\prime \prime \star}$ was 
Table 4. Estimated parameters from Schemes 2, 3, and 4 fitted to $\alpha 1 \mathrm{~K} 276 \mathrm{E} \beta$ glycine receptor single-channel data

\begin{tabular}{|c|c|c|c|}
\hline & Scheme 2: flip & $\begin{array}{l}\text { Scheme 3: full } \\
\text { primed }\end{array}$ & $\begin{array}{l}\text { Scheme 4: reduced } \\
\text { primed }\end{array}$ \\
\hline$\alpha_{1}^{\prime}\left(s^{-1}\right)$ & $4340 \pm 23 \%$ & $47,000 \pm 61 \%$ & \\
\hline$\beta_{1}^{\prime}\left(s^{-1}\right)$ & $13,600 \pm 20 \%$ & $12,000 \pm 48 \%$ & \\
\hline$\alpha_{2}^{\prime}\left(s^{-1}\right)$ & $18,500 \pm 16 \%$ & $9530 \pm 94 \%$ & $13,600 \pm 15 \%$ \\
\hline$\beta_{2}^{\prime}\left(s^{-1}\right)$ & $16,300 \pm 26 \%$ & $410 \pm 59 \%$ & $596 \pm 27 \%$ \\
\hline$\alpha_{3}^{\prime}\left(s^{-1}\right)$ & $31,200 \pm 6 \%$ & $13,700 \pm 12 \%$ & $14,900 \pm 36 \%$ \\
\hline$\beta_{3}^{\prime}\left(s^{-1}\right)$ & $85,600 \pm 2 \%$ & $1260 \pm 20 \%$ & $1720 \pm 21 \%$ \\
\hline$\alpha_{2}^{\prime \prime}\left(s^{-1}\right)$ & & $26,200 \pm 83 \%$ & \\
\hline$\beta_{2}^{\prime \prime}\left(s^{-1}\right)$ & & $2340 \pm 86 \%$ & \\
\hline$\alpha_{3}^{\prime \prime}\left(s^{-1}\right)$ & & $9100 \pm 27 \%$ & $5570 \pm 7 \%$ \\
\hline$\beta_{3}^{\prime \prime}\left(s^{-1}\right)$ & & $57,000 \pm 18 \%$ & $44,300 \pm 8 \%$ \\
\hline$\alpha_{3}^{\prime \prime \prime}\left(s^{-1}\right)$ & & $27,600 \pm 7 \%$ & $26,700 \pm 12 \%$ \\
\hline$\beta_{3}^{\prime \prime \prime}\left(s^{-1}\right)$ & & $119,000 \pm 2 \%$ & $116,000 \pm 3 \%$ \\
\hline$\gamma_{1}^{\prime}\left(s^{-1}\right)$ & $19,000 \pm 6 \%$ & $11,900 \pm 27 \%$ & \\
\hline$\delta_{1}^{\prime}\left(s^{-1}\right)$ & $34 \pm 22 \%$ & $92 \pm 61 \%$ & \\
\hline$\gamma_{2}^{\prime}\left(s^{-1}\right)$ & $727,000 \pm 19 \%$ & $38,200 \pm 41 \%$ & $8900 \pm 10 \%$ \\
\hline$\delta_{2}^{\prime}\left(s^{-1}\right)$ & $17,300 \pm 21 \%$ & $1160 \pm 13 \%$ & $1410 \pm 19 \%$ \\
\hline$\gamma_{3}^{\prime}\left(s^{-1}\right)$ & $281 \pm 55 \%$ & $1550 \pm 7 \%$ & $2050 \pm 4 \%$ \\
\hline$\delta_{3}^{\prime}\left(s^{-1}\right)$ & $100 \pm 55 \%$ & $545 \pm 8 \%$ & $547 \pm 6 \%$ \\
\hline$\gamma_{2}^{\prime \prime}\left(s^{-1}\right)$ & & $447,000 \pm 17 \%$ & \\
\hline$\delta_{2}^{\prime \prime}\left(s^{-1}\right)$ & & $532,000 \pm 35 \%$ & \\
\hline$\gamma_{3}^{\prime \prime}\left(s^{-1}\right)$ & & $2710 \pm 51 \%$ & $19,000 \pm 8 \%$ \\
\hline$\delta_{3}^{\prime \prime}\left(s^{-1}\right)$ & & $946 \pm 45 \%$ & $5640 \pm 13 \%$ \\
\hline$\gamma_{3}^{\prime \prime \prime}\left(s^{-1}\right)$ & & $1600 \pm 7 \%$ & $1430 \pm 16 \%$ \\
\hline$\delta_{3}^{\prime \prime \prime}\left(s^{-1}\right)$ & & $728 \pm 15 \%$ & $770 \pm 11 \%$ \\
\hline$k_{-}\left(s^{-1}\right)$ & $230 \pm 22 \%$ & $161 \pm 53 \%$ & $110 \pm 38 \%$ \\
\hline$k_{+}\left(M^{-1} s^{-1}\right)$ & $7.34 \times 10^{4} \pm 34 \%$ & $5.57 \times 10^{4} \pm 54 \%$ & $5.10 \times 10^{4} \pm 39 \%$ \\
\hline$k_{-}^{\prime}\left(s^{-1}\right)$ & $1080 \pm 3 \%$ & $4290 \pm 22 \%$ & $241,000 \pm 34 \%$ \\
\hline$k_{+}^{\prime}\left(M^{-1} S^{-1}\right)$ & $4.61 \times 10^{6} \pm 27 \%$ & $1.93 \times 10^{6} \pm 63 \%$ & $2.05 \times 10^{8} \pm 43 \%$ \\
\hline$k_{-}^{\prime \prime}\left(s^{-1}\right)$ & & $6660 \pm 16 \%$ & \\
\hline$k_{+}^{\prime \prime}\left(\mathrm{M}^{-1} \mathrm{~s}^{-1}\right)$ & & $7.67 \times 10^{6} \pm 35 \%$ & \\
\hline$E_{1}^{\prime}=\beta_{1}^{\prime} / \alpha_{1}^{\prime}$ & $3.19 \pm 4 \%$ & $0.275 \pm 62 \%$ & \\
\hline$E_{2}^{\prime}=\beta_{2}^{\prime} / \alpha_{2}^{\prime}$ & $1.00 \pm 43 \%$ & $0.123 \pm 85 \%$ & $0.044 \pm 19 \%$ \\
\hline$E_{3}^{\prime}=\beta_{3}^{\prime} / \alpha_{3}^{\prime}$ & $2.76 \pm 4 \%$ & $0.0968 \pm 28 \%$ & $0.212 \pm 64 \%$ \\
\hline$E_{2}^{\prime \prime}=\beta_{2}^{\prime \prime} / \alpha_{2}^{\prime \prime}$ & & $0.270 \pm 98 \%$ & \\
\hline$E_{3}^{\prime \prime}=\beta_{3}^{\prime \prime} / \alpha_{3}^{\prime \prime}$ & & $6.69 \pm 12 \%$ & $8.07 \pm 13 \%$ \\
\hline$E_{3}^{\prime \prime \prime}=\beta_{3}^{\prime \prime \prime} / \alpha_{3}^{\prime \prime \prime}$ & & $4.37 \pm 7 \%$ & $4.47 \pm 10 \%$ \\
\hline$F_{1}^{\prime}=\delta_{1}^{\prime} / \gamma_{1}^{\prime}$ & $0.00177 \pm 16 \%$ & $0.00606 \pm 51 \%$ & \\
\hline$F_{2}^{\prime}=\delta_{2}^{\prime} / \gamma_{2}^{\prime}$ & $0.0236 \pm 4 \%$ & $0.0419 \pm 34 \%$ & $0.157 \pm 12 \%$ \\
\hline$F_{3}^{\prime}=\delta_{3}^{\prime} / \gamma_{3}^{\prime}$ & $0.325 \pm 10 \%$ & $0.352 \pm 6 \%$ & $0.266 \pm 2 \%$ \\
\hline$F_{2}^{\prime \prime}=\delta_{2}^{\prime \prime} / \gamma_{2}^{\prime \prime}$ & & $1.27 \pm 33 \%$ & \\
\hline$F_{3}^{\prime \prime}=\delta_{3}^{\prime \prime} / \gamma_{3}^{\prime \prime}$ & & $0.352 \pm 21 \%$ & $0.297 \pm 11 \%$ \\
\hline$F_{3}^{\prime \prime \prime}=\delta_{3}^{\prime \prime \prime} / \gamma_{3}^{\prime \prime \prime}$ & & $0.465 \pm 20 \%$ & $0.551 \pm 7 \%$ \\
\hline$K_{\mathrm{r}}=k_{-} / k_{+}(\mu \mathrm{M})$ & $3690 \pm 27 \%$ & $3130 \pm 11 \%$ & $2220 \pm 5 \%$ \\
\hline$K^{\prime}=k_{-}^{\prime} / k_{+}^{\prime}(\mu \mathrm{M})$ & $271 \pm 25 \%$ & $376 \pm 32 \%$ & $1340 \pm 18 \%$ \\
\hline$K^{\prime \prime}=k_{-}^{\prime \prime} / k_{+}^{\prime \prime}(\mu \mathrm{M})$ & & $1110 \pm 31 \%$ & \\
\hline $\max P_{\text {open }}$ & $0.488 \pm 11 \%$ & $0.420 \pm 10 \%$ & $0.411 \pm 9 \%$ \\
\hline$E C_{50}(\mu \mathrm{m})$ & $9170 \pm 24 \%$ & $6300 \pm 15 \%$ & $4900 \pm 8 \%$ \\
\hline$n_{\mathrm{H}}$ & $1.34 \pm 2 \%$ & $1.40 \pm 0.4 \%$ & $1.35 \pm 1 \%$ \\
\hline
\end{tabular}

Rate constants are means of fits to three data sets, each containing observations at four glycine concentrations \pm the coefficient of variation of the mean. Equilibrium constants $(E, F$, and $K)$, the average $\max P_{\text {open }}, E C_{50}$, and $n_{\mathrm{H}}$ are the mean values of the three values calculated for each separate data set \pm the coefficient of variation of the mean. Schemes 2, 3, and 4 have 14,27, and 19 free parameters, respectively. All three schemes in this table were constrained by the assumption that the binding sites are equivalent and independent. This constraint was implemented by the following equations: $k_{+1}=k_{+2}=k_{+3}=k_{+}$and $k_{-1}=k_{-2}=k_{-3}=k_{-}$. In addition, one rate constant in each cycle was fixed by the constraint of microscopic reversibility (Colquhoun et al., 2004).

always $<0.3 \%$, and that of the shut states $A F^{\prime}, A_{2} F^{\prime}$, and $A_{2} F^{\prime \prime}$ was invariably $<2 \%$, even at $300 \mu \mathrm{M}$ glycine. The relative frequency with which these six states were visited was assessed from the equilibrium initial vector for openings and its analog for shuttings (Colquhoun and Hawkes, 1982, Equations 3.63 and 3.90, respectively). These initial vectors give the relative frequency of transitions from shut states to each open state and from open states to each shut state. It does not include transitions between shut states that have no direct connection with an open state. The six states we examined were predicted to be visited relatively infrequently at all concentrations, but omitting all six did not give good fits. Therefore, a reduced primed model was defined by omitting only open states $A F^{\prime *}$ and $A_{2} F^{\prime \prime \star}$ and shut states $A F^{\prime}$ and $A_{2} F^{\prime \prime}$. The retained singly primed states, $A_{2} F^{\prime \star}$ and $A_{2} F^{\prime}$, had a somewhat higher occupancy and frequency of visitation at higher concentrations, and were needed for a good fit (next section), but there is an arbitrary element in their choice.

\section{Scheme 4: "reduced primed" mechanism}

The "reduced primed" mechanism (Fig. 7A, Scheme 4) is a subset of the full primed mechanism where the states rarely visited in $\alpha 1 \mathrm{~K} 276 \mathrm{E} \beta$ are removed. As seen from the summary in Table 4 (third column), the fits with Scheme 4 were very consistent and the majority of rate constants had low coefficients of variation. The least well defined rate constants were those defining binding/ unbinding steps. An example of a fit to one dataset is shown in Figure 7, $B$ and $C$. The quality of predictions was very good for all plots (open and shut time distributions, the negative correlation between open and shut time duration at high glycine, and the $P_{\text {open }}$-concentration curve) and very nearly as good as with Scheme 3, despite considerably fewer free parameters (19, compared with 27 for Scheme 3).

Opening rate constants increased with increasing level of ligation and priming (Table 4; Fig. 8). Although fits of Scheme 4 suggest that the true mean open time is not very concentration dependent, the apparent mean open time increases with concentration (Fig. $2 B$, empirical fits). The fit predicts that at $300 \mu \mathrm{M}$ glycine, apparent openings quite often started in a diliganded open state $[43 \%$ start this way, based on the averaged HawkesJalali-Calquhoun initial vector], whereas at $100 \mathrm{~mm}$ glycine only $0.2 \%$ of apparent openings start in a diliganded state.

Fits of both Scheme 3 and Scheme 4 (see Table 4) suggest that the opening rate constant of the fully liganded, fully primed receptor is very similar to the fully liganded opening rate constant from the flip mechanism in wild-type channels (compare 116,000 for Scheme 4 to $129,000 \mathrm{~s}^{-1}$ in Burzomato et al., 2004). The efficacy of this opening, however, may be lower than that for the wild type because of a higher closing rate constant $(26,700$ vs $\left.7000 \mathrm{~s}^{-1}\right)$. Although these rate constants are found by fitting different subsets of the same (primed) model, they are intended to represent the same physical event and should therefore be comparable. As in wild-type channels, in the mutant the values of the closing rates were not particularly sensitive to the degree of flipping/priming, suggesting that once the channel has opened, its stability is relatively uniform, regardless of what conformational changes have preceded the opening.

The fully liganded flipping equilibrium constant is 27 in wildtype heteromeric receptors, but in the mutant all three fully liganded priming equilibrium constants were much lower, in the range of 0.2 to 0.5 , similar to partially liganded flipping constants in wild-type channels ( 0.4 for diliganded receptors). The effective efficacy of fully liganded mutant receptors was 0.64 , which is considerably lower than that of wild-type receptors $\left(E_{\mathrm{eff}}=19\right)$.

A prominent property of the flip mechanism fit to wild-type heteromeric glycine receptors is that agonist-binding affinity is about 65 times higher for the flipped conformation than for the resting conformation. This affinity increase was almost absent in the K276E mutant, being less than twofold. Glycine affinity for the resting conformation of the mutant receptor $(\sim 2 \mathrm{mM})$ was only about four times lower than for the wild-type receptor $(520 \mu \mathrm{M})$. The 
A

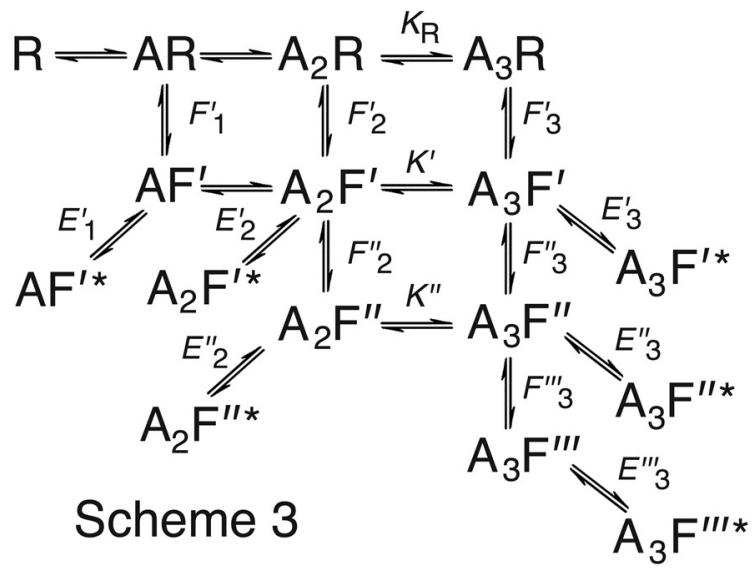

B

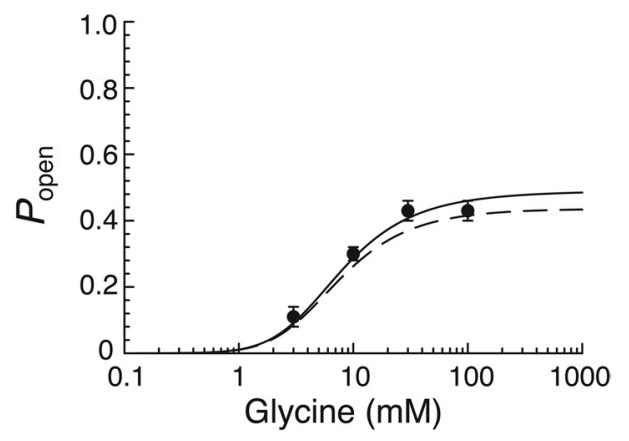

C
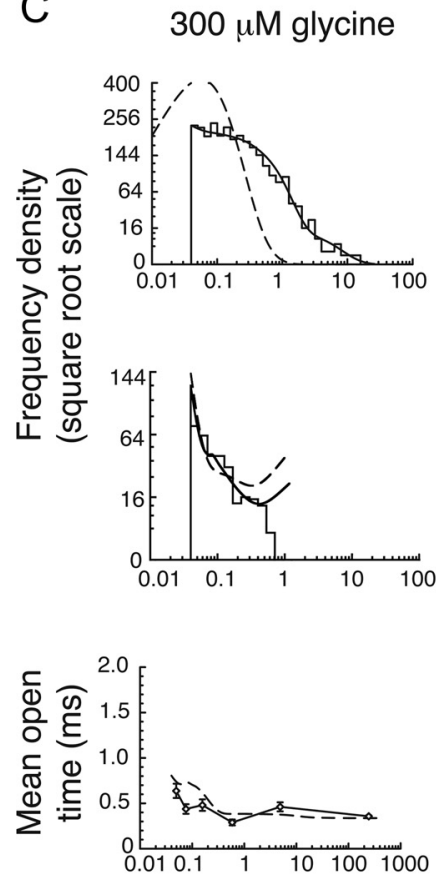

$10 \mathrm{mM}$ glycine

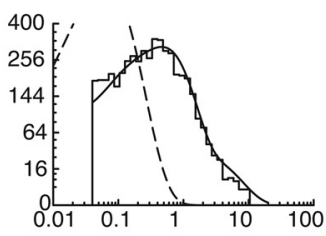

Apparent open periods (ms)
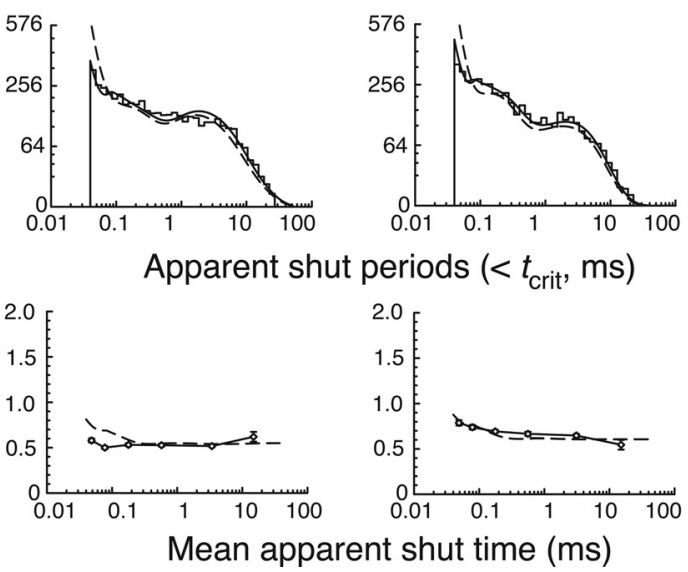

$100 \mathrm{mM}$ glycine
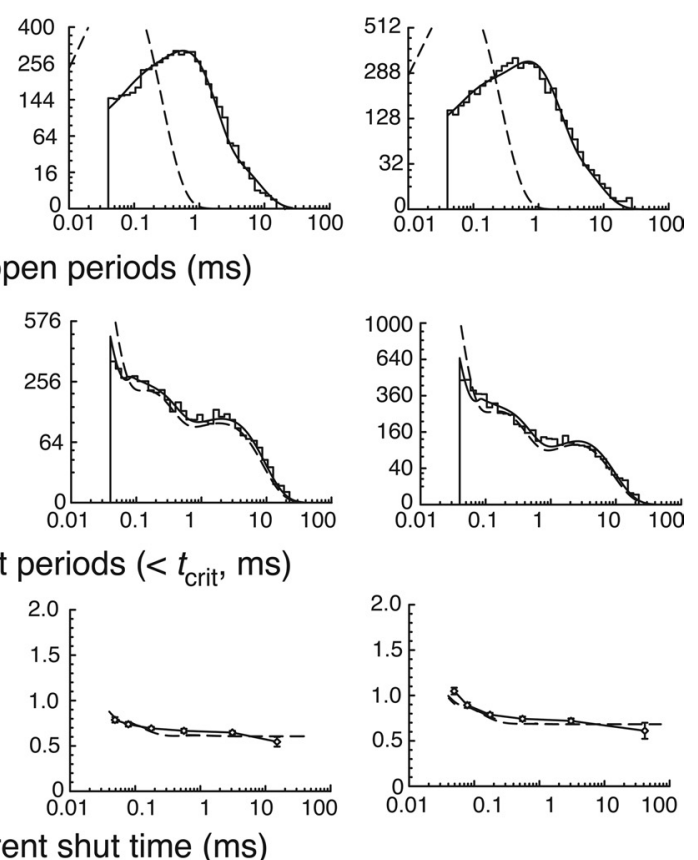

Figure 6. Fits of a primed mechanism to the heteromeric mutant receptor single-channel data. $\boldsymbol{A}$, Scheme 3 is a primed mechanism adapted from Mukhtasimova et al. (2009). Six open states (asterisks) can be accessed from an array of different ligation and different level of primed shut states. This mechanism has 27 free parameters after imposing constraints similar to those for $S c h e m e$ 2. $B, C$, The results of Scheme 3 fit to the same dataset as in Figures 4 and 5 . For the details, see Figure 4 . Note that the predictions of the open period distributions, the shut time distributions, and the correlation plots were very good at all concentrations. The $P_{\text {open }}$ curve was also predicted well. Average rate constants are given in Table 4.

rate constant for dissociation from the fully liganded singly primed conformation is estimated to be $k_{-}^{\prime}=241,000 \mathrm{~s}^{-1}$ (Table 4). This is almost certainly too fast to be estimated accurately. Inspection of the covariance matrix shows that there is a very strong correlation between the dissociation and association rate constants (range $r=0.92-0.98$ for the three datasets). This means that their ratio $K^{\prime}=k_{-}^{\prime} / k_{+}^{\prime}=1340 \mu \mathrm{M}$ is much better determined than the separate rate constants. The same is true for binding to the resting conformation. All we can say is that the rates for binding to the primed conformation appear to be fast.

Attempts to fit the primed mechanism to wild-type channels We tried several variants of Scheme 4 on wild-type heteromeric channels (data not shown). The results were inconsistent from one set to another (the one exception being the opening rate constant, which again came out consistently very fast, 166,000 $\mathrm{s}^{-1}$ ), probably because the wild-type channel shows no sign of more than one open time component at saturating concentration (Table 1, Figs. 1 and $8 B$ in Burzomato et al., 2004). In contrast, the mutant receptor clearly has at least three open time components when saturated (Fig. 2; Table 1). This means that, for the wild-type receptor, there is very little information about intermediates other than a single flip state even for the saturated receptor. A further problem is that, for fits to K276E, we pruned Scheme 3 to reduce the number of free parameters in the mechanism by removing low-liganded states that were rarely visited and had brief lifetimes. In contrast, the wild-type receptor opened frequently when partially liganded (Burzomato et al., 2004).

In summary, with currently available data, the mutant and wild-type receptors clearly need to be fitted with different subsets of the full primed model. Qualitatively, the mutation had its greatest effect in reducing the efficacy of the preopening flipping/ priming steps, whereas the final gating steps are similar for wild- 
A

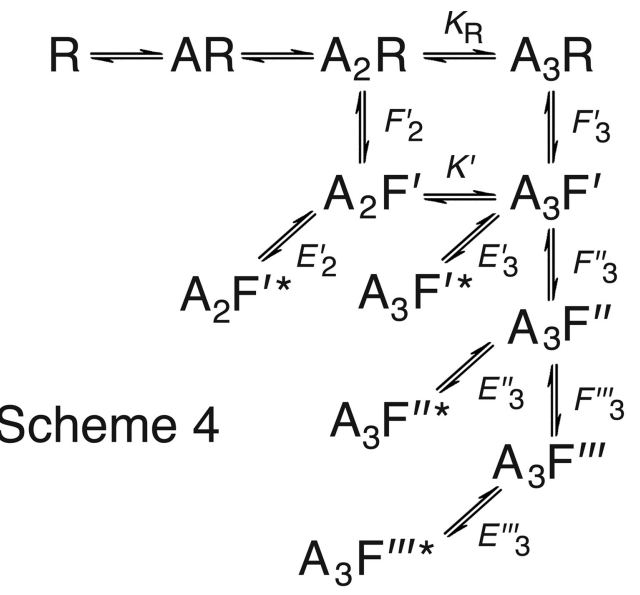

C

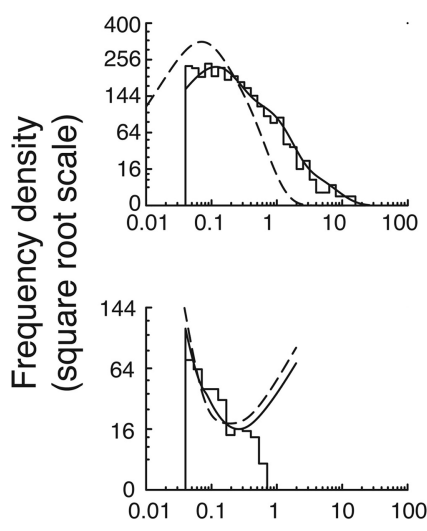

$10 \mathrm{mM}$ glycine
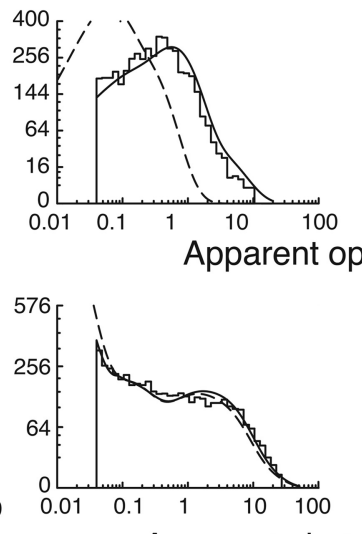

Apparent shut

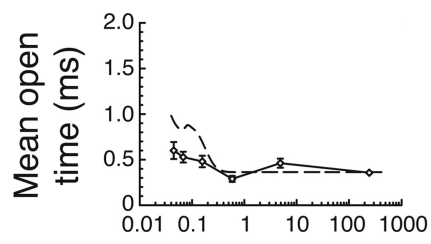

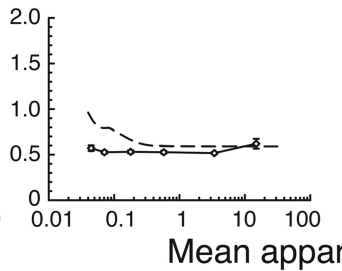

B

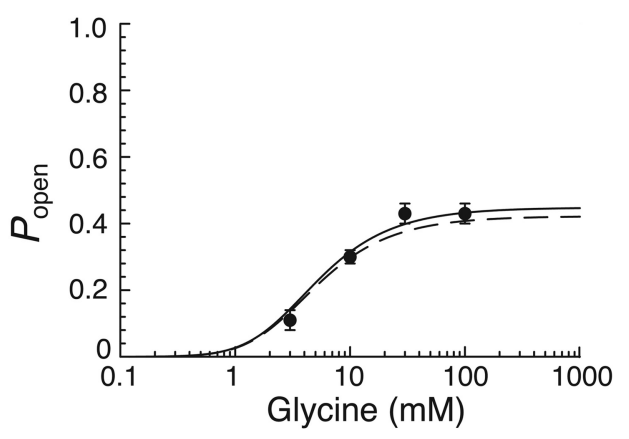

$30 \mathrm{mM}$ glycine

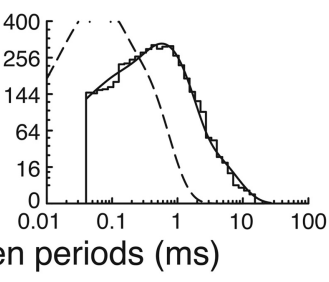

$100 \mathrm{mM}$ glycine
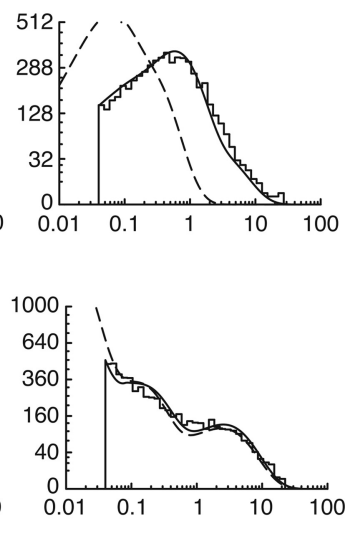

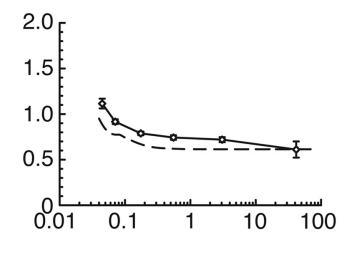

Figure 7. Fits of a reduced primed mechanism to the heteromeric mutant receptor single-channel data. $\boldsymbol{A}$, Scheme 4 is a subset of the primed mechanism. To obtain it, states with low occupancy were removed from $S$ cheme 3. For fitting, constraints similar to those used for $S$ cheme 2 were imposed. As a consequence, the reduced primed mechanism has 19 free parameters, fewer than those for the full primed mechanism. B, C, The results of Scheme 4 fit to the same dataset as in Figures 4, 5, and 6 . For the details, see Figure 4 . The fits of Scheme 4 were almost as good as with Scheme 3 despite many fewer free parameters. Table 4 gives average rate constants.

type and mutant. This mirrors the situation for full and partial agonists (Lape et al., 2008), for the A52S mutant (Plested et al., 2007), and for spontaneous versus liganded openings in ACh muscle nicotinic receptors (Mukhtasimova et al., 2009).

\section{Burst length distribution}

The ability to predict the observed distribution of duration of groups of openings ("bursts") provides another test of the fitted mechanism. The definition of "bursts" raises some interesting problems, which will be described.

In records at $300 \mu \mathrm{M}$ glycine, the lowest concentration at which channel activity was observed, the shut time distribution had a minimum at approximately $1 \mathrm{~ms}$ (Fig. $2 B$ ), and this led us to impose a critical time interval of around $1 \mathrm{~ms}$, to divide records into bursts. The representative burst length distribution plotted in Figure $9 A$ ( $t_{\text {crit }}$ of $0.6 \mathrm{~ms}$ ) shows a mixture of four exponential components (the longest of which was minimal in area). Observed bursts were brief (mean length, $0.36 \mathrm{~ms}$ ) and most had only a single opening, as the main component in the distribution of number of open periods per burst had a mean of $1.09 \pm 0.03$ open periods (area, $74 \pm 6 \%, n=4$; data not shown).

In contrast, the theoretical burst length distribution (calculated from the fit of Scheme 4, Fig. 9B, dashed line; Eq. 3.17 in Colquhoun and Hawkes, 1982) is predicted to contain, along with short bursts, many longer ones ( $18.3 \mathrm{~ms}, 39 \%$ of area; $49 \mathrm{~ms}, 54 \%$ of area). It is important to determine whether this discrepancy is a symptom of the inadequacy of Scheme 4 or whether it stems from other factors, such as distortions introduced by a poor experimental choice of $t_{\text {crit }}$ or by the finite recording bandwidth.

A tractable form for open time within a burst, when bursts are defined by an arbitrary $t_{\text {crit }}$, has been reported (Ball et al., 2007), but the equivalent expression for burst duration is not available. Instead, we simulated a record from one set of the fitted rate constants for Scheme 4 at $300 \mu \mathrm{M}$ glycine and imposed a realistic $40 \mu$ s temporal resolution. We plotted the burst length distribution for a $t_{\text {crit }}$ of $0.6 \mathrm{~ms}$, the same as for experimental burst length distri- 
bution. The distribution so obtained (Fig. 9B, histogram and continuous line fit) is similar to the experimental burst length distribution. Scheme 4, therefore, predicts the burst length successfully.

This apparent discrepancy in Figure $9 B$ is easily explained. The theoretical bursts (Fig. 9B, dashed line) were calculated on the basis that the end of a burst was demarcated by at least one sojourn in state $R$ (Fig. 8, State 12). Any time spent continuously within shut states 5-11 was counted as being "within bursts." This definition of a burst is appropriate if we want to know about individual receptor activations, defined as the visible part of what happens between the binding of the first agonist molecule and the dissociation of the last agonist molecule (Edmonds et al., 1995). The mechanism predicts that the bursts themselves are sparse groups of openings with low open probability (5\% at $300 \mu \mathrm{M}$ glycine), and that the overall $P_{\text {open }}$ (e.g., bursts together with the interburst intervals) is $1.9 \%$. The bursts contain shut times that are quite long $(6.1 \mathrm{~ms}$, $24 \%$ area; $32 \mathrm{~ms}, 2.1 \%$ area). These longer shut times are excluded by a $t_{\text {crit }}$ of $0.6 \mathrm{~ms}$. To include shuttings of this length within bursts, a much longer $t_{\text {crit }}$ would be needed, and that would mean using a still lower concentration of agonist (or patches with only one channel). Either approach would necessitate recording for many hours to collect enough openings. This is not possible with an average patch lifetime of $\sim 15 \mathrm{~min}$.

The reason for using such a short $t_{\text {crit }}$ is that it produces a clear demarcation between groups of openings (Fig. $2 \mathrm{~B}$, distribution of shut times). The $P_{\text {open }}$ within such groups is high enough that we can be sure that the openings within them originate from one individual channel. These criteria are necessary and sufficient considerations for fitting with HJCFIT.

At a low concentration like $300 \mu \mathrm{M}$ glycine, the time spent in state $A R$ (Fig. 8, state 11 ; within the theoretical burst) is quite long (predicted mean life, $30 \mathrm{~ms}$ ), because of the slow rate of association at low agonist concentration. In fact, if we define shut times within bursts to include only the flipped states (Fig. 8, shut states 5-8), the mean predicted burst length is only $0.39 \mathrm{~ms}$, similar to the mean for observed bursts defined with a $t_{\text {crit }}$ of $0.6 \mathrm{~ms}$ (Fig. $9 A ; 0.42 \mathrm{~ms}$, Fig. $9 B$ ). This procedure excludes from bursts sojourns in the compound shut state $(A R$, $A_{2} R, A_{3} R$; states 9-11), sojourns that have a mean duration of $26 \mathrm{~ms}$.

\section{Concentration jumps: a comparison between experimental responses and those predicted by simulations}

Wyllie et al. (1998) demonstrated that the time constants of the low-concentration burst length distribution reflect the deactivation time constants observed in relaxations to zero agonist con- centration. To understand the mutant channel behavior in nonequilibrium conditions, we used outside-out patches to record macroscopic currents activated by brief pulses of saturating glycine (Fig. 10A). The onset of wild-type heteromeric responses was fast (gray trace, $0.21 \pm 0.01 \mathrm{~ms} ; 10-90 \%$ rise time; $n=8$ patches) and possibly limited by solution exchange. The black trace in Figure $10 \mathrm{~A}$ shows clearly that mutant heteromeric receptors had a much slower rising phase. The $10-90 \%$ rise times were on average approximately sevenfold slower than wild type ( $1.5 \pm 0.2 \mathrm{~ms}, n=5$; Fig. 10C).

Deactivation, the decay of patch currents after removal of glycine, was, unlike rise times, much faster for mutant than for wild-type receptors. The deactivation decay of mutant channels 
A

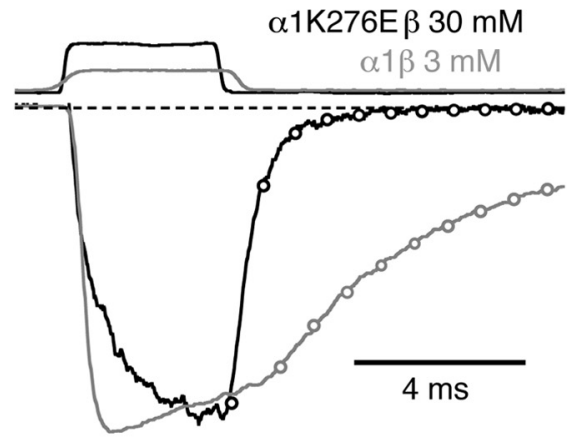

B

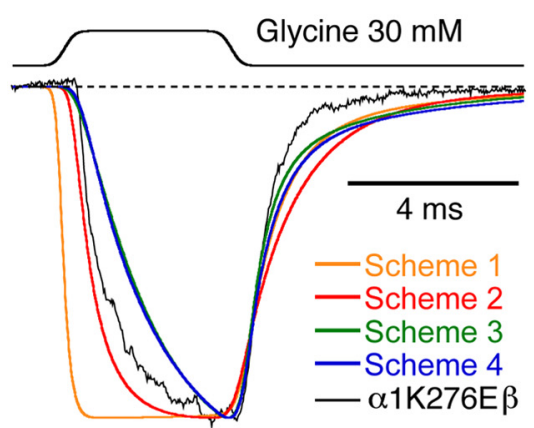

C

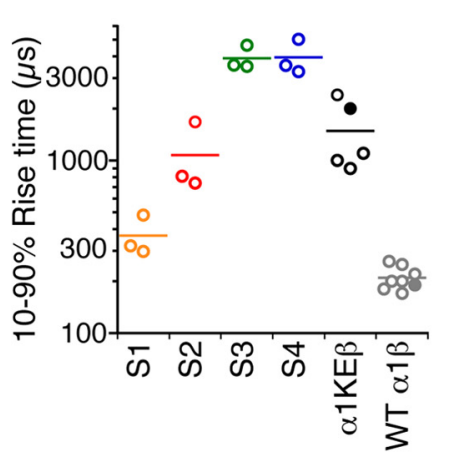

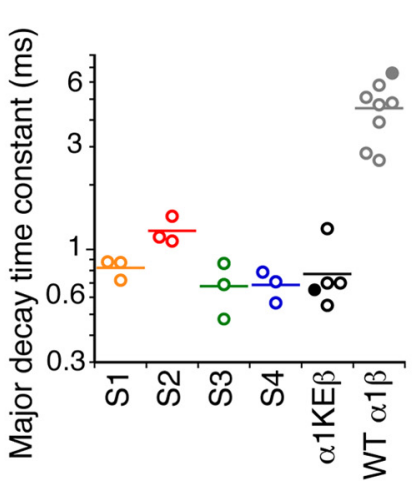

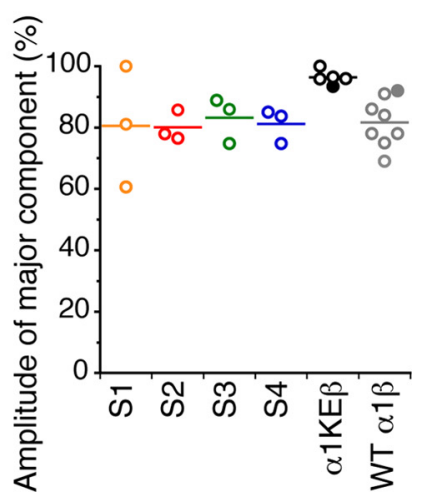

Figure 10. Comparison of empirical and predicted concentration jumps. $A$, The rise time of wild-type $\alpha 1 \beta$ glycine receptor response to 3 mm glycine is extremely fast (lower gray trace, $10-90 \%$ rise time of $190 \mu \mathrm{s}$ for the trace shown). The decay of the response after the end of the pulse was much slower, and well fitted by three exponential components [open gray circles, $\tau_{1}=6.6 \mathrm{~ms}$ (amplitude $92 \%$ ), $\tau_{2}=30 \mathrm{~ms}(7 \%)$, and $\tau_{3}=149 \mathrm{~ms}(1 \%)$ for this record]. The rising phase of the response of $\alpha 1 \mathrm{KE} \beta$ receptors (lower black trace) to a $\sim 4 \mathrm{~ms}$ pulse of $30 \mathrm{~mm}$ glycine was, on average, sevenfold slower than wild type. In multiple patches, the response apparently failed to peak during a $\sim 3 \mathrm{~ms}$ pulse, but in other patches, the responses were faster. The decay of the $\alpha 1 \mathrm{KE} \beta$ current following the removal of glycine was rapid, and well described by a double exponential function. The patch shown here was fit with time constants of $0.65 \mathrm{~ms}(94 \%)$ and $3.9 \mathrm{~ms}$. The upper traces show the solution exchanges measured at the open tip at the end of each experiment (black for K276E, gray for wild type). B, Superimposition of a representative experimental current trace (black) with predicted responses to a $4 \mathrm{~ms}$ pulse of $30 \mathrm{~mm}$ glycine from the four mechanisms fitted to single-channel data. The predicted traces are the averages of the responses calculated from each set of fitted rate constants. Calculated responses were normalized to the peak before averaging. Scheme 1 (orange trace) predicts a much faster rise time than observed for experimental jumps. Scheme 2 (red trace) predicts a slightly faster rise time. Both Schemes 3 and 4 (green and blue traces, respectively) predicted slow-rising phases that failed to peak during the 4 ms pulse. C, Dot plots summarizing the $10-90 \%$ rise times and the major decay time constant $\left(\tau_{1}\right)$ and its amplitude $\left(A_{1}\right)$ for responses calculated from the fitted mechanisms and experimental jumps (color coding as in $\boldsymbol{B}$, wild-type values in gray). The values for each fitted dataset are shown, and the mean is indicated as a bar. In experimental jumps on the K276E mutant (black symbols), only a very small slow component of the decay was detected. For all the mechanisms tested, this slow component was predicted to have, on average, about $20 \%$ of the total amplitude. Filled circles represent the values for the experimental traces plotted in $\boldsymbol{A}$ and $\boldsymbol{B}$.

was best fitted with two exponentials, whereas wild-type decays needed three components. Time constants (and relative amplitudes at $t=0)$ were $0.75 \pm 0.09 \mathrm{~ms}(96.5 \pm 0.9 \%)$ and $11 \pm 4 \mathrm{~ms}$ $(3.5 \pm 0.9 \% ; n=7$ patches) for mutant receptors and $4.5 \pm 0.5$ $\mathrm{ms}(82 \pm 3 \%), 23 \pm 3 \mathrm{~ms}(16 \pm 3 \%)$, and $170 \pm 30 \mathrm{~ms}(1.9 \pm$ $0.4 \% ; n=8)$ for wild-type receptors.

Next, we checked how well our fitted mechanisms from the single-channel data could predict the time course of the observed macroscopic currents. We took the fitted rate constants and calculated macroscopic responses to concentration jumps with a realistic solution exchange (see Materials and Methods for details; $4-12 \mathrm{~ms}$ pulses of $30 \mathrm{~mm}$ glycine with $10-90 \%$ rise and fall times of $500 \mu \mathrm{s})$. Normalized calculated currents are shown in Figure $10 B$ together with a representative experimental response. All schemes predicted a deactivation time course that was reasonably close to the experimental observations, but they diverged in their ability to describe the current rise time (Fig. 10C). Scheme 1 failed to predict the slow rising phase of mutant channels. Despite failing to describe several aspects of the single-channel data, Scheme 2 (flip) gave the best prediction of the observed rise time (at the same time, the description of the decay phase by Scheme 2 was marginally worse than that by the other schemes; Fig. 10C). The rise times of the currents simulated from the primed schemes
(Schemes 3 and 4) were somewhat slower than the responses of the mutant receptor (Fig. 10C, left).

The deactivation of macroscopic currents calculated from the results of the single-channel analysis had two components in a range likely to be detectable. The dominant fast deactivation time constant predicted by Schemes 2-4 was very close to that observed in experimental jumps (Fig. 10C, middle and right). However, all mechanisms predicted also a slower component. This was most pronounced for Scheme 2. For Scheme 4, the slower component $(4.8 \pm 0.5 \mathrm{~ms}$ ) was small (amplitude at $t=0,19 \pm$ $4 \%$ ) but consistent. Examination of the occupancy of individual open states during the decay suggests that this component derives from the fully primed open state, which is the most likely to be visited on multiple occasions. A similar slow component was detected in the experimental responses to concentration jumps but was much smaller (3.5\%).

\section{Discussion}

Direct detection of multiple short-lived intermediates in the gating pathway

The K276E mutation lies in the M2-M3 loop between the agonist-binding domain and the channel gate. Given that it is in the "gating pathway," and probably moves as the channel opens, 
it is not surprising that the charge change from lysine to glutamate strongly affects activation. There is a 30 -fold increase in glycine $E C_{50}$ in mutant heteromers, and this is largely due to an impairment in gating. The affinity of glycine for the resting state (according to our mechanisms fitted by maximum likelihood) is not greatly reduced by the K276E mutation (e.g., Schemes 3 and $4, K=3.1 \mathrm{~mm}$ and $2.2 \mathrm{~mm}$, respectively; compare with Burzomato et al., 2004, $K=0.520 \mathrm{~mm}$ for wild type). Schild analysis with the competitive antagonist strychnine also suggests that the binding site itself is quite similar in wild-type and $\mathrm{K} 276 \mathrm{E}$ mutant receptors (Lewis et al., 1998).

The conformational changes that occur before the channel opening are much weaker in the mutant: they do not increase glycine affinity much. In the wild type, there is a 65 -fold increase in affinity with flipping (Burzomato et al., 2004). This greater affinity after flipping is mainly due to a faster association rate constant, and it drives closed bound receptors toward the open state and increases the lifetime of the bound complex. For K276E receptors, this intermediate state has much lower affinity so the average affinity of the receptor for glycine (across all states) is reduced, resulting in a low potency for activation.

Recordings from mutant channels have several distinctive features that reflect the presence of multiple steps between the resting and open conformations. At maximally effective glycine concentrations, heteromeric wild-type receptors have high open probability and the distributions of shut times become quite simple: the vast majority of shuttings are brief. Heteromeric K276E receptors instead exhibit a wide range of shut time durations across the entire concentration range. This suggests the presence of several intermediate shut states between resting and open conformations, as specified by the primed schemes, 3 and 4 . This is why these schemes describe our recordings much better than the flip mechanism, which has only one intermediate shut state. According to this interpretation, the K276E mutation "reveals" additional minima in the energy landscape, in which wild-type receptors dwell too briefly for the sojourns to be detected at our resolution.

Further, the rising phase of responses to a jump into saturating glycine is substantially slowed in the K276E mutant, consistent with the expected delay caused by slow transit through intermediate priming states.

In addition to that, at saturating glycine, the distribution of apparent open times is complex for $\mathrm{K} 276 \mathrm{E}$ receptors. Wild-type channels do not deviate noticeably from a monoexponential open time distribution, consistent with a single fully liganded open state. In contrast to that, the saturated mutant receptor clearly has more than one exponential in the apparent open time distribution, which suggests that there is more than one fully liganded open state. This explicit feature of the priming mechanisms allows channels to open when only some of the subunits are in the primed state (Mukhtasimova et al., 2009).

Critically, even when the receptor is saturated by glycine (100 $\mathrm{mm}$ ), adjacent open and shut intervals exhibited a moderate yet robust negative correlation. Such a correlation is not detected in saturated wild-type channels (Burzomato et al., 2004). This observation places strong constraints on the topology of any mechanism proposed to describe the data (Colquhoun and Hawkes, 1987). A linear sequence of shut and open states would predict no correlations. The observed correlation is unlikely to result from distortions introduced by finite time resolution (Ball and Sansom, 1988). In fact, missing brief events as a consequence of limited time resolution may tend to obscure such correlations (Schorge et al., 2005).
In conclusion, the mutant channel is better fitted by mechanisms that include multiple priming steps rather than a concerted flip. The final "fast" gating step seen in wild-type receptors is not altered much by this mutation, just as it is similar for weak agonists and strong ones (Lape et al., 2008). The final open-shut step seems to be a fast and stereotyped reaction little affected by changes of structure (in either the agonist or in the receptor) that have been studied to date.

The K276E mutation slows the passage of the receptor through serial preopen steps, allowing the channel to open from partially activated intermediates. Our calculations suggest that the mean latency to opening, starting from the fully liganded resting state, is $5.3 \pm 0.4 \mathrm{~ms}$ for the mutant (for Scheme 4 ; compared to $0.061 \mathrm{~ms}$ for the wild-type flip mechanism). We propose that in wild-type receptors, this passage is so fast that it is not distinguishable from the concerted flipping that we have previously used in our mechanisms. What are the real conformational changes behind these states? Mechanisms in which each binding site, located $\sim 50 \AA$ from the others, is included as an independent entity that does not interact with the other sites, describe both glycine and nicotinic channel activity well. It is appealing to extend this idea to the conformational changes before the stereotyped opening of the channel gate.

The priming steps in the mechanism proposed by Mukhtasimova et al. (2009), on the basis of crosslinking experiments, describe nonconcerted, independent changes that follow the initial rearrangement at each binding domain. In the M2-M3 loop at the interface of the extracellular and transmembrane domains, the K276E mutation may act by interfering with conformational changes distinct from those at the binding site. In this view, the mutation might have uncovered a cascade of motions similar to that proposed for muscle nicotinic receptors (Auerbach, 2010). However, the correlations between open and shut sojourns that we observe are compatible only with a receptor that can open from the intermediate states in this cascade.

\section{Gating of homomeric K276E receptors}

Strikingly, at all concentrations up to $200 \mathrm{~mm}$, activations of K276E homomeric mutant channels appeared uniformly spaced, without obvious clustering, and thus differed from other glycine receptors that we have examined, such as $\alpha 1, \alpha 2, \alpha 1 \beta$, and $\alpha 1$ A52S $\beta$ (Beato et al., 2004; Burzomato et al., 2004; Plested et al., 2007; Krashia et al., 2011). Another difference was that the pattern of activations appeared to be extremely weakly dependent on concentration. This limited the usefulness of the recordings for direct fitting. Our observations nonetheless suggest two mechanistic principles underlying the activation of the homomeric K276E mutant: (1) the open lifetime must be short; and (2) reopening after channel closure should be much less likely than agonist dissociation. In this interpretation, increasing the agonist concentration merely increases the frequency of brief individual openings. Unfortunately, without methods to count channels in a cell-attached patch, we are unable to measure this frequency.

\section{A role for the $\boldsymbol{\beta}$ subunit in channel gating?}

Homomeric receptors, which harbor five mutation copies, have severely impaired gating, whereas heteromeric mutant receptors reach half the open probability of wild-type channels when saturated by glycine. This disparity suggests two possibilities. First, the number of copies of the mutation, regardless of the presence of $\beta$ subunits, might determine the degree of gating impairment. An alternative, intriguing possibility is that the $\beta$ subunit plays a role in gating of the receptor. In this interpretation, the $\beta$ subunit 
boosts the gating of the receptor, leading to a partial rescue of the gating behavior. A corollary of this idea is that mutations in the $\beta$ subunit, distant from the channel gate and not immediately within the binding site, should alter receptor activity in the same way as mutations in the $\alpha$ subunit. The existence of a set of mutants in the $\beta$ subunit that shift $E C_{50}$ was previously taken to indicate that the $\beta$ subunit forms the principal binding subunit in heteromeric channels (Grudzinska et al., 2005). The strong rescue of gating that we observe upon inclusion of the $\beta$ subunit suggests that this idea may require some revision.

\section{The synaptic impairment in K276E mutant receptors}

For the first time, our measurements enable us to estimate the likely degree of impairment produced by a hyperekplexia mutation at a human synapse. The glycine concentration profile at mammalian synapses has been estimated to reach a peak of 2.2$3.5 \mathrm{~mm}$, which then decays with a time constant of $0.6-0.9 \mathrm{~ms}$ (Beato, 2008). The mutant channel response to a synaptic-like pulse of glycine ( $1 \mathrm{~ms}, 3 \mathrm{~mm}$ ) would be $>100$-fold smaller than that of wild type on average, reaching a maximum $P_{\text {open }}$ of $0.3 \%$ versus $81 \%$. It is of course possible that in patients who are mostly heterozygotes synaptic receptors contain a mixture of mutant and wild-type $\alpha$ subunits and are therefore less impaired in gating.

The K276E hyperekplexia mutation in the glycine receptor proves once again that, by selecting functionally important mutations, human channelopathies can be surprisingly powerful tools to reveal channel mechanisms (Engel et al., 2010). This hyperekplexia mutation slows what are normally very fast steps in receptor gating. Future studies of glycine receptor gating may take advantage of K276E as a "slow motion" background mutation, to unmask details of channel activation usually beyond the resolution of our technique. Thus, this mutation may also prove a useful addition to the channel biophysicist's protein engineering toolbox (Jadey et al., 2011).

\section{References}

Auerbach A (2010) The gating isomerization of acetylcholine receptors. J Physiol 588:573-586.

Ball FG, Sansom MS (1988) Single-channel autocorrelation functions: the effects of time interval omission. Biophys J 53:819-832.

Ball FG, Milne RK, Yeo GF (2007) Marked continuous-time Markov chain modelling of burst behaviour for single ion channels. J Appl Math Decis Sci 2007: Article ID 48138. doi:10.1155/2007/48138.

Beato M (2008) The time course of transmitter at glycinergic synapses onto motoneurons. J Neurosci 28:7412-7425.

Beato M, Groot-Kormelink PJ, Colquhoun D, Sivilotti LG (2002) Openings of the rat recombinant $\alpha 1$ homomeric glycine receptor as a function of the number of agonist molecules bound. J Gen Physiol 119:443-466.

Beato M, Groot-Kormelink PJ, Colquhoun D, Sivilotti LG (2004) The activation of $\alpha 1$ homomeric glycine receptors. J Neurosci 24:895-906.

Benndorf K (1995) Low-noise recording. In: Single-channel recording (Sakmann B, Neher E, eds), pp 129-153. New York: Plenum.

Bormann J, Rundström N, Betz H, Langosch D (1993) Residues within transmembrane segment M2 determine chloride conductance of glycine receptor homo- and hetero-oligomers. EMBO J 12:3729-3737.

Burzomato V, Groot-Kormelink PJ, Sivilotti LG, Beato M (2003) Stoichiometry of recombinant heteromeric glycine receptors revealed by a porelining region point mutation. Receptors Channels 9:353-361.

Burzomato V, Beato M, Groot-Kormelink PJ, Colquhoun D, Sivilotti LG (2004) Single-channel behavior of heteromeric $\alpha 1 \beta$ glycine receptors: an attempt to detect a conformational change before the channel opens. J Neurosci 24:10924-10940.

Chakrapani S, Bailey TD, Auerbach A (2004) Gating dynamics of the acetylcholine receptor extracellular domain. J Gen Physiol 123:341-356.

Chung SK, Vanbellinghen JF, Mullins JG, Robinson A, Hantke J, Hammond CL, Gilbert DF, Freilinger M, Ryan M, Kruer MC, Masri A, Gurses C,
Ferrie C, Harvey K, Shiang R, Christodoulou J, Andermann F, Andermann E, Thomas RH, Harvey RJ, et al. (2010) Pathophysiological mechanisms of dominant and recessive GLRA1 mutations in hyperekplexia. J Neurosci 30:9612-9620.

Colquhoun D, Hawkes AG (1977) Relaxation and fluctuations of membrane currents that flow through drug-operated channels. Proc R Soc Lond B Biol Sci 199:231-262.

Colquhoun D, Hawkes AG (1982) On the stochastic properties of bursts of single ion channel openings and of clusters of bursts. Philos Trans R Soc Lond B Biol Sci 300:1-59.

Colquhoun D, Hawkes AG (1987) A note on correlations in single ion channel records. Proc R Soc Lond B Biol Sci 230:15-52.

Colquhoun D, Hawkes AG (1990) Stochastic properties of ion channel openings and bursts in a membrane patch that contains two channels: evidence concerning the number of channels present when a record containing only single openings is observed. Proc R Soc Lond B Biol Sci 240:453-477.

Colquhoun D, Sigworth FJ (1995) Fitting and statistical analysis of singlechannel records. In: Single-channel recording (Sakmann B, Neher E, eds), pp 483-587. New York: Plenum.

Colquhoun D, Hawkes AG, Srodzinski K (1996) Joint distributions of apparent open and shut times of single-ion channels and maximum likelihood fitting of mechanisms. Philos Trans R Soc Lond A 354:2555-2590.

Colquhoun D, Hatton CJ, Hawkes AG (2003) The quality of maximum likelihood estimates of ion channel rate constants. J Physiol 547:699-728.

Colquhoun D, Dowsland KA, Beato M, Plested AJ (2004) How to impose microscopic reversibility in complex reaction mechanisms. Biophys J 86:3510-3518.

Edmonds B, Gibb AJ, Colquhoun D (1995) Mechanisms of activation of muscle nicotinic acetylcholine receptors, and the time course of endplate currents. Annu Rev Physiol 57:469-493.

Elmslie FV, Hutchings SM, Spencer V, Curtis A, Covanis T, Gardiner RM, Rees M (1996) Analysis of GLRA1 in hereditary and sporadic hyperekplexia: a novel mutation in a family cosegregating for hyperekplexia and spastic paraparesis. J Med Genet 33:435-436.

Engel AG, Shen XM, Selcen D, Sine SM (2010) What have we learned from the congenital myasthenic syndromes. J Mol Neurosci 40:143-153.

Gill SB, Veruki ML, Hartveit E (2006) Functional properties of spontaneous IPSCs and glycine receptors in rod amacrine (AII) cells in the rat retina. J Physiol 575:739-759.

Groot-Kormelink PJ, Beato M, Finotti C, Harvey RJ, Sivilotti LG (2002) Achieving optimal expression for single channel recording: a plasmid ratio approach to the expression of $\alpha 1$ glycine receptors in HEK293 cells. J Neurosci Methods 113:207-214.

Grosman C (2003) Free-energy landscapes of ion-channel gating are malleable: changes in the number of bound ligands are accompanied by changes in the location of the transition state in acetylcholine-receptor channels. Biochemistry 42:14977-14987.

Grosman C, Zhou M, Auerbach A (2000) Mapping the conformational wave of acetylcholine receptor channel gating. Nature 403:773-776.

Grudzinska J, Schemm R, Haeger S, Nicke A, Schmalzing G, Betz H, Laube B (2005) The $\beta$ subunit determines the ligand binding properties of synaptic glycine receptors. Neuron 45:727-739.

Jadey SV, Purohit P, Bruhova I, Gregg TM, Auerbach A (2011) Design and control of acetylcholine receptor conformational change. Proc Natl Acad Sci U S A 108:4328-4333.

Jones MV, Westbrook GL (1995) Desensitized states prolong GABA A $_{\text {chan- }}$ nel responses to brief agonist pulses. Neuron 15:181-191.

Krashia P, Lape R, Lodesani F, Colquhoun D, Sivilotti LG (2011) The long activations of $\alpha 2$ glycine channels can be described by a mechanism with reaction intermediates ("flip"). J Gen Physiol 137:197-216.

Langosch D, Laube B, Rundström N, Schmieden V, Bormann J, Betz H (1994) Decreased agonist affinity and chloride conductance of mutant glycine receptors associated with human hereditary hyperekplexia. EMBO J 13:4223-4228.

Lape R, Colquhoun D, Sivilotti LG (2008) On the nature of partial agonism in the nicotinic receptor superfamily. Nature 454:722-727.

Lape R, Krashia P, Colquhoun D, Sivilotti LG (2009) Agonist and blocking actions of choline and tetramethylammonium on human muscle acetylcholine receptors. J Physiol 587:5045-5072.

Legendre P, Muller E, Badiu CI, Meier J, Vannier C, Triller A (2002) Desen- 
sitization of homomeric $\alpha 1$ glycine receptor increases with receptor density. Mol Pharmacol 62:817-827.

Lewis TM, Sivilotti LG, Colquhoun D, Gardiner RM, Schoepfer R, Rees M (1998) Properties of human glycine receptors containing the hyperekplexia mutation $\alpha 1(\mathrm{~K} 276 \mathrm{E})$, expressed in Xenopus oocytes. J Physiol 507:25-40.

Meyer G, Kirsch J, Betz H, Langosch D (1995) Identification of a gephyrin binding motif on the glycine receptor $\beta$ subunit. Neuron 15:563-572.

Moroni M, Biro I, Giugliano M, Vijayan R, Biggin PC, Beato M, Sivilotti LG (2011) Chloride ions in the pore of glycine and GABA channels shape the time course and voltage dependence of agonist currents. J Neurosci 31:14095-14106.

Mukhtasimova N, Lee WY, Wang HL, Sine SM (2009) Detection and trapping of intermediate states priming nicotinic receptor channel opening. Nature 459:451-454.

Pitt SJ, Sivilotti LG, Beato M (2008) High intracellular chloride slows the decay of glycinergic currents. J Neurosci 28:11454-11467.

Plested AJ, Groot-Kormelink PJ, Colquhoun D, Sivilotti LG (2007) Single channel study of the spasmodic mutation $\alpha 1 \mathrm{~A} 52 \mathrm{~S}$ in recombinant rat glycine receptors. J Physiol 581:51-73.

Pribilla I, Takagi T, Langosch D, Bormann J, Betz H (1992) The atypical M2 segment of the $\beta$ subunit confers picrotoxinin resistance to inhibitory glycine receptor channels. EMBO J 11:4305-4311.

Sachs F (1999) Practical limits on the maximal speed of solution exchange for patch clamp experiments. Biophys J 77:682-690.

Schorge S, Elenes S, Colquhoun D (2005) Maximum likelihood fitting of single channel NMDA activity with a mechanism composed of independent dimers of subunits. J Physiol 569:395-418.

Sivilotti LG (2010) What single channel analysis tells us of the activation mechanism of ligand-gated channels: the case of the glycine receptor. J Physiol 588:45-58.

Thompson AJ, Lester HA, Lummis SC (2010) The structural basis of function in Cys-loop receptors. Q Rev Biophys 43:449-499.

Wyllie DJ, Béhé P, Colquhoun D (1998) Single-channel activations and concentration jumps: comparison of recombinant NR1a/NR2A and NR1a/NR2D NMDA receptors. J Physiol 510:1-18. 